\title{
ON THE SCATTERING OF LONGITUDINAL ELASTIC WAVES FROM AXISYMMETRIC DEFECTS IN COATED PIPES
}

\author{
Ray Kirby* \\ School of Engineering and Design, \\ Mechanical Engineering, \\ Brunel University, \\ Uxbridge, Middlesex, UB8 3PH, UK. \\ ray.kirby@brunel.ac.uk
}

Zahari Zlatev

School of Engineering and Design,

Mechanical Engineering, Brunel University, Uxbridge, Middlesex, UB8 3PH, UK.

\author{
Peter Mudge \\ NDT Technology Group \\ TWI Ltd \\ Cambridge CB21 6AL, UK
}

Keywords: Elastic waves, coated pipes, mode matching, hybrid finite element method Running Title: sound scattering in coated pipes

* Corresponding author 


\begin{abstract}
Viscoelastic coatings are widely used to protect pipelines from their surrounding environment. These coatings are known to attenuate ultrasonic waves guided along the pipe walls, which may limit the range of a pulse/echo based inspection technique that seeks to detect defects in a pipeline. This article aims to investigate the attenuation of longitudinal modes in a coated pipe by comparing predicted and measured values for the reflection coefficient of an axisymmetric defect in a pipe coated with bitumen. This extends recent work undertaken by the authors for torsional modes, and also provides an independent investigation into the validity of those values proposed by the authors for the shear properties of bitumen, based on a comparison between prediction and experiment for torsional modes. Predictions are generated using a numerical mode matching approach for axially uniform defects, and a hybrid finite element based method for non-uniform defects. Values for the shear and longitudinal properties of bitumen are investigated and it is shown that the shear properties of the viscoelastic material play a dominant role in the propagation of longitudinal modes in a coated pipeline. Moreover, by using the shear values obtained from experiments on torsional modes, it is shown that good agreement between prediction and measurement for uniform and nonuniform defects may also be obtained for the longitudinal $\mathrm{L}(0,2)$ mode. This provides further validation for the shear bulk acoustic properties proposed for bitumen in the low ultrasonic frequency range, although in order to apply this methodology in general it is demonstrated that one must measure independently the reflection coefficient of both the torsional $T(0,1)$ and the longitudinal $\mathrm{L}(0,2)$ mode before arriving at values for the shear properties of a viscoelastic material.
\end{abstract}




\section{INTRODUCTION}

This article is intended to be a companion to an article published by the same authors on ultrasonic torsional wave mode propagation in coated pipes [1]. For torsional modes it was shown that by using the semi analytic finite element (SAFE) method to find the eigenmodes for a coated pipe, and then combining this with a mode matching or hybrid finite element based technique, one may successfully predict the reflection coefficient for an axisymmetric defect. Moreover, by isolating the refection of the $\mathrm{T}(0,1)$ mode from a uniform axisymmetric defect in a coated pipe, it is possible to infer values for the bulk shear properties of the viscoelastic coating by comparing predicted and measured reflection coefficients. Here, the values obtained by Kirby et al. [1] for the shear attenuation coefficient of bitumen were shown to be different from those values published in the literature by Barshinger and Rose [2] and Simonetti and Cawley [3]. Those values found by Kirby et al. [1] were obtained using a trial and error process in which the relevant viscoelastic parameters in the theoretical model were changed until good agreement between predicted and measured data was obtained. Whilst this method was shown to be capable of delivering good agreement between prediction and measurement for $\mathrm{T}(0,1)$, the viscoelastic parameters obtained will also play a role in the propagation of longitudinal modes. That is, those properties obtained by trial and error for the $\mathrm{T}(0,1)$ modes will not necessarily deliver good agreement for an independent set of experimental measurements undertaken using L $(0,2)$ modes. Accordingly, this article compares experimental measurements against predictions obtained for $\mathrm{L}(0,2)$ modes, generated using those viscoelastic properties found when studying $\mathrm{T}(0,1)$ modes [1].

The difficulty of independently obtaining data for the shear bulk acoustic properties of a viscoelastic material in the low ultrasonic frequency range has encouraged the use of guided wave based methods. Typically one attempts to infer the viscoelastic properties from the measurement of, for example, modal phase speed or attenuation. This type of approach was used by Simonetti 
and Cawley [3], who examined the refection of the $\mathrm{T}(0,1)$ mode from a solid disc of bitumen sandwiched between two stainless steel rods. Simonetti and Cawley were able to obtain values for the shear attenuation and velocity for bitumen; however, the method does depend on the availability of a solid disc, or cylinder, of material in bulk form, which is not always possible for those viscoelastic materials used to coat pipelines. Moreover, the quality of the data obtained by Simonetti and Cawley will also depend upon maintaining continuity conditions at the interface between the disc and the two adjoining rods. Ma et al. [4] also used torsional modes to examine the scattering from a relatively thick layer of epoxy placed inside a pipe. They measured a change in the cut-on frequencies of the higher order torsional modes when a material was present, and from this were able to determine the bulk shear velocity of the epoxy layer. A similar technique was also implemented by Castaings and Bacon [5], who used the finite element method to generate predictions for a pipe filled with bitumen, which were then compared against experimental measurements in order to infer values of the Lame constant $\mu$ for bitumen. The study of Castaings and Bacon used the commercial code COMSOL to generate the predictions and so the focus was on generating those constants required by the commercial software, rather than looking at the constituent components of $\mu$. The method of Castaings and Bacon does, however, require the use of large amounts of bitumen sufficient to fill a hollow tube, which may prove difficult to realise for some coating materials. In view of this, the original purpose of the study by Kirby et al. [1] was to use guided waves to investigate the properties of a viscoelastic material coating the walls of a pipe. This permits the study of many different types of coating without requiring them in bulk form. This method also permits the isolation of the shear wave speed as well as the shear attenuation for the viscoelastic material. It is, however, interesting to note that the method of Kirby et al [1], as well as those other methods used on pipes, have been restricted to the study of torsional modes. This is, perhaps, not surprising given the interest in the shear properties, although it is well known that the propagation of longitudinal modes is also influenced by these shear properties. Accordingly, the influence of a viscoelastic coating on the propagation of longitudinal modes is studied here. 
In LRUT it is common to use longitudinal modes, either as an alternative to torsional modes, or as an additional set of tests. The $\mathrm{L}(0,2)$ mode is typically chosen for these tests because it is relatively non-dispersive over a normal LRUT frequency range of approximately 20 to $120 \mathrm{kHz}$; the $\mathrm{L}(0,2)$ mode is also the fastest propagating mode and this makes it easier to separate from other reflected modes [6]. Thus, commercial equipment such as the Teletest device [7] used by Kirby et al. [1] is designed to measure $\mathrm{L}(0,2)$ modes as well as torsional modes, and so it is relatively straightforward to repeat the tests in [1] using longitudinal modes. The presence of a coating will, of course, serve to attenuate the longitudinal modes in the same way as that seen for the torsional modes and this will impact on the ability to locate defects in coated pipelines. Nevertheless, it is important first to identify satisfactorily the viscoelastic properties before attempting to use a theoretical model to optimise LRUT in coated pipes.

It is known that the shear properties of a viscoelastic material play a significant role in the propagation of longitudinal modes [8]. However, very few studies have investigated the influence of a viscoelastic coating on the propagation of longitudinal modes in pipes. Barshinger and Rose [2] did measure the longitudinal bulk acoustic properties for bitumen, and then compared the measured attenuation of the $\mathrm{L}(0,1), \mathrm{L}(0,2)$ and $\mathrm{L}(0,3)$ modes against analytic predictions. However, the experimental measurements of Barshinger and Rose are largely limited to above 0.6 $\mathrm{MHz}$, with only a few data points around 0.2 MHz. The analysis of Barshinger and Rose [2] has been extended to make use of the SAFE method, see for example Marzani et al [9], and Hua and Rose [10]; however, there is little further experimental data available in the literature with which to validate predictions obtained using these models, especially at lower frequencies. Accordingly, there is a clear need to review and further validate the viscoelastic properties obtained using shear/torsional modes against data measured separately for longitudinal modes, especially in the frequency range 20 to $120 \mathrm{kHz}$. 
The methodology used by Kirby et al. [1] to study the reflection of the $\mathrm{T}(0,1)$ mode from uniform and non-uniform defects in coated pipes is adopted here for longitudinal modes. That is, a mode matching approach suitable for uniform defects is examined first, followed by a hybrid numerical method suitable for studying non-uniform defects. These numerical methods are well documented in the literature and a brief review was provided in [1], therefore these methods will not be discussed further here. However, for completeness, relevant examples of the SAFE method may be found in refs. [11-15], for the mode matching method in [16-18], and for the hybrid method [1922]. Here, the numerical methods applied to elastic wave propagation are generally based on the use of commercial software, which causes some problems when it comes to identifying the appropriate viscoelastic material properties suitable for the generic mass and stiffness matrices typically specified by commercial codes. To overcome this potential problem, the analysis that follows focuses on axisymmetric modes only, as this facilitates the separation of the torsional and longitudinal modes. This avoids the need to sort these modes from an unordered list of axisymmetric and non-axisymmetric modes (typical in commercial software), which is especially important when studying coated pipes where such a sorting procedure is likely to be very computationally expensive. Thus, in section 2.1 the SAFE method is applied to longitudinal modes only; these modes are then used in a mode matching method for uniform defects in section 2.2, and a hybrid method for non-uniform defects is presented in section 2.3. Following a brief discussion of the experimental methodology in section 3, predictions are then compared against measurements in section 4 .

\section{THEORY}


The propagation of elastic waves in a pipe is governed by Navier's equation,

$$
\mu_{\mathrm{p}, \mathrm{c}} \nabla^{2} \mathbf{u}^{\prime}+\left(\lambda_{\mathrm{p}, \mathrm{c}}+\mu_{\mathrm{p}, \mathrm{c}}\right) \nabla\left(\nabla \cdot \mathbf{u}^{\prime}\right)=\frac{\partial^{2} \mathbf{u}^{\prime}}{\partial t^{2}}
$$

where $\mu$ and $\lambda$ are the Lamé constants, $\mathbf{u}^{\prime}$ is the displacement vector and $t$ is time. It is assumed here that the pipe consists of a steel substrate, in which sound attenuation may be neglected, which is coated externally by a viscoelastic material in which sound attenuation is assumed to take place. Here, the steel pipe is denoted by subscript $p$, and the external coating by subscript c. It is convenient to use a cylindrical co-ordinate system $(r, \theta$ and $z)$, and for an axisymmetric problem this delivers three equations, only two of which are coupled. The two coupled equations (displacements in the $r$ and $z$ direction) govern the propagation of the longitudinal pipe modes to be examined here, whereas the third equation (displacement in the $\theta$ direction) governs the propagation of the torsional modes. The focus of this article is on the longitudinal modes and here Navier's equation reduces to two (coupled) scalar equations [23]:

$$
\frac{\partial^{2} u^{\prime}}{\partial r^{2}}+\frac{1}{r} \frac{\partial u^{\prime}}{\partial r}-\frac{u^{\prime}}{r^{2}}+\frac{c_{\mathrm{p}, \mathrm{c}}^{2}}{a_{\mathrm{p}, \mathrm{c}}^{2}} \frac{\partial^{2} u^{\prime}}{\partial z^{2}}+\frac{\partial}{\partial r}\left(\frac{\partial w^{\prime}}{\partial z}\right)-\frac{c_{\mathrm{p}, \mathrm{c}}^{2}}{a_{\mathrm{p}, \mathrm{c}}^{2}} \frac{\partial}{\partial z}\left(\frac{\partial w^{\prime}}{\partial r}\right)=\frac{1}{a_{\mathrm{p}, \mathrm{c}}^{2}} \frac{\partial^{2} u^{\prime}}{\partial t^{2}}
$$

and

$$
\frac{\partial^{2} w^{\prime}}{\partial r^{2}}+\frac{1}{r} \frac{\partial w^{\prime}}{\partial r}+\frac{a_{\mathrm{p}, \mathrm{c}}^{2}}{c_{\mathrm{p}, \mathrm{c}}^{2}} \frac{\partial^{2} w^{\prime}}{\partial z^{2}}+\left[\frac{a_{\mathrm{p}, \mathrm{c}}^{2}}{c_{\mathrm{p}, \mathrm{c}}^{2}}-1\right] \frac{1}{r}\left(\frac{\partial u^{\prime}}{\partial z}\right)+\frac{a_{\mathrm{p}, \mathrm{c}}^{2}}{c_{\mathrm{p}, \mathrm{c}}^{2}} \frac{\partial}{\partial z}\left(\frac{\partial u^{\prime}}{\partial r}\right)-\frac{\partial}{\partial r}\left(\frac{\partial u^{\prime}}{\partial z}\right)=\frac{1}{c_{\mathrm{p}, \mathrm{c}}^{2}} \frac{\partial^{2} w^{\prime}}{\partial t^{2}}
$$

where $u^{\prime}$ and $w^{\prime}$ are the displacements in the $r$ and $z$ directions, respectively. The torsional and longitudinal bulk wave velocities are denoted by $c$ and $a$, respectively.

\subsection{Eigenvalue Analysis}


An eigenvalue problem is obtained by expanding the displacements $u^{\prime}$ and $w^{\prime}$ over the pipe eigenmodes to give

$$
u^{\prime}(r, z ; t)=\sum_{n=0}^{\infty} u^{n}(r) \mathrm{e}^{i\left[k_{\mathrm{p}} \lambda^{n} z-\omega t\right]}
$$

and

$$
w^{\prime}(r, z ; t)=\sum_{n=0}^{\infty} w^{n}(r) \mathrm{e}^{i\left[k_{\mathrm{p}} \lambda^{n} z-\omega t\right]}
$$

where $u^{n}(r)$ and $w^{n}(r)$ are the eigenvectors for the coated pipe, $k_{\mathrm{p}}=\omega / c_{\mathrm{p}}$ so that $\lambda^{n}$ is a dimensionless wavenumber, $t$ is time, $\omega$ is the radian frequency, and $i=\sqrt{-1}$. The eigenvalue analysis is applied to a pipe with a steel substrate of inner radius $a_{1}$ and outer radius $b_{1}$ (this is region $\mathrm{p}$, with a substrate wall thickness $t_{\mathrm{p}}=b_{1}-a_{1}$ ), and a pipe coating of inner radius $b_{1}$ and outer radius $b_{2}$ (this is region c, with a coating thickness $t_{\mathrm{c}}=b_{2}-b_{1}$ ). The substitution of Eqs. (4) and (5) into Eqs. (2) and (3) yields, for any mode n,

$$
\frac{\partial^{2} u_{\mathrm{p}, \mathrm{c}}}{\partial r^{2}}+\frac{1}{r} \frac{\partial u_{\mathrm{p}, \mathrm{c}}}{\partial r}-\frac{u_{\mathrm{p}, \mathrm{c}}}{r^{2}}-\frac{c_{\mathrm{p}, \mathrm{c}}^{2}}{a_{\mathrm{p}, \mathrm{c}}^{2}} k_{\mathrm{p}}^{2} \lambda^{2} u_{\mathrm{p}, \mathrm{c}}+\gamma_{\mathrm{p}, \mathrm{c}}^{2} u_{\mathrm{p}, \mathrm{c}}+i k_{\mathrm{p}} \lambda\left[1-\frac{c_{\mathrm{p}, \mathrm{c}}^{2}}{a_{\mathrm{p}, \mathrm{c}}^{2}}\right] \frac{\partial w_{\mathrm{p}, \mathrm{c}}}{\partial r}=0
$$

and

$$
\frac{\partial^{2} w_{\mathrm{p}, \mathrm{c}}}{\partial r^{2}}+\frac{1}{r} \frac{\partial w_{\mathrm{p}, \mathrm{c}}}{\partial r}-\frac{a_{\mathrm{p}, \mathrm{c}}^{2}}{c_{\mathrm{p}, \mathrm{c}}^{2}} k_{\mathrm{p}}^{2} \lambda^{2} w_{\mathrm{p}, \mathrm{c}}+\gamma_{\mathrm{p}, \mathrm{c}}^{2} w_{\mathrm{p}, \mathrm{c}}+i k_{\mathrm{p}} \lambda\left[\frac{a_{\mathrm{p}, \mathrm{c}}^{2}}{c_{\mathrm{p}, \mathrm{c}}^{2}}-1\right]\left(\frac{u_{\mathrm{p}, \mathrm{c}}}{r}+\frac{\partial u_{\mathrm{p}, \mathrm{c}}}{\partial r}\right)=0,
$$

with $\gamma_{\mathrm{p}, \mathrm{c}}=\omega / a_{\mathrm{p}, \mathrm{c}}$. The appropriate radial boundary conditions on the inner and outer walls are

$$
T_{r r_{\mathrm{p}}}=0 \text { and } T_{r z_{\mathrm{p}}}=0 \text { at } r=a_{1} \text {; and } T_{r r_{\mathrm{c}}}=0 \text {, and } T_{r z_{\mathrm{c}}}=0 \text { at } r=b_{2} \text {, }
$$

where $T$ is the shear stress, with $T_{r z}=T_{z r}$, so that

and

$$
\frac{T_{r r_{\mathrm{p}, \mathrm{c}}}}{\rho_{\mathrm{p}, \mathrm{c}} a_{\mathrm{p}, \mathrm{c}}^{2}}=\frac{\partial u_{\mathrm{p}, \mathrm{c}}}{\partial r}-\left[\frac{2 c_{\mathrm{p}, \mathrm{c}}^{2}}{a_{\mathrm{p}, \mathrm{c}}^{2}}-1\right]\left(\frac{u_{\mathrm{p}, \mathrm{c}}}{r}+i k_{\mathrm{p}} \lambda w_{\mathrm{p}, \mathrm{c}}\right)
$$




$$
\frac{T_{r z_{\mathrm{p}, \mathrm{c}}}}{\rho_{\mathrm{p}, \mathrm{c}} c_{\mathrm{p}, \mathrm{c}}^{2}}=i k_{\mathrm{p}} \lambda u_{\mathrm{p}, \mathrm{c}}+\frac{\partial w_{\mathrm{p}, \mathrm{c}}}{\partial r}
$$

Here, $\rho_{\mathrm{p}, \mathrm{c}}$ is the density of the steel pipe (p) or coating (c). At the interface between the pipe substrate and coating, continuity of shear stress and displacement is enforced, so at $r=b_{1}$

$$
T_{r r_{\mathrm{p}}} \cdot \mathbf{n}_{\mathrm{p}}=T_{r r_{\mathrm{c}}} \cdot \mathbf{n}_{\mathrm{c}} \text { and } T_{r Z_{\mathrm{p}}} \cdot \mathbf{n}_{\mathrm{p}}=T_{r z_{\mathrm{c}}} \cdot \mathbf{n}_{\mathrm{c}}
$$

and

$$
u_{\mathrm{p}}=u_{\mathrm{c}} \text { and } w_{\mathrm{p}}=w_{\mathrm{c}}
$$

Here $\mathbf{n}$ is an (outward) unit normal. A standard finite element analysis is adopted here so that the displacements are discretised to give

$$
u(r)=\sum_{j=1}^{p_{2}} N_{j}(r) u_{j}
$$

and

$$
w(r)=\sum_{j=1}^{p_{2}} M_{j}(r) w_{j}
$$

where $N_{j}$ and $M_{j}$ are global trial (or shape) functions for the (transverse) one dimensional finite element mesh; $u_{j}$ and $w_{j}$ are the values of $u(r)$ and $w(r)$ at node $j$, respectively, and $p_{2}$ is the number of nodes (or degrees of freedom) lying on the pipe cross section $a_{1} \leq r \leq b_{2}$. Expressing Eqs. (13) and (14) in vector form [1] yields $u(r)=\mathbf{N u}$ and $w(r)=\mathbf{M w}$, where column vectors $\mathbf{u}$ and $\mathbf{w}$ have length $p_{2}$. Following Kirby $[1,12]$, the boundary conditions at the walls of the pipe are first introduced to Eqs. (6) and (7) and then the equations for the coated and uncoated regions are joined using Eqs. (11) and (12). This generates two coupled equations, which in matrix form yields

$$
\mathbf{R}_{0} \mathbf{u}+\lambda \mathbf{Z}_{1} \mathbf{w}+\lambda^{2} \mathbf{R}_{2} \mathbf{u}=\mathbf{0}
$$


and

$$
\mathbf{Z}_{0} \mathbf{w}+\lambda \mathbf{R}_{1} \mathbf{u}+\lambda^{2} \mathbf{Z}_{2} \mathbf{w}=\mathbf{0},
$$

where $\mathbf{u}=\left[\begin{array}{ll}\mathbf{u}_{\mathrm{p}} & \mathbf{u}_{\mathrm{c}}\end{array}\right]$ and $\mathbf{w}=\left[\begin{array}{ll}\mathbf{w}_{\mathrm{p}} & \mathbf{w}_{\mathrm{c}}\end{array}\right]$. In addition

$$
\mathbf{R}_{q}=\mathbf{R}_{q}^{\mathrm{p}} \mathbf{u}_{\mathrm{p}}+\sigma \mathbf{R}_{q}^{\mathrm{c}} \mathbf{u}_{\mathrm{c}}
$$

and

$$
\mathbf{Z}_{q}=\mathbf{Z}_{q}^{\mathrm{p}} \mathbf{u}_{\mathrm{p}}+\beta \mathbf{Z}_{q}^{\mathrm{c}} \mathbf{u}_{\mathrm{c}}
$$

for $q=0,1$ and 2, with $\sigma=\rho_{\mathrm{c}} a_{c}^{2} / \rho_{\mathrm{p}} a_{\mathrm{p}}^{2}$ and $\beta=\rho_{\mathrm{c}} c_{c}^{2} / \rho_{\mathrm{p}} c_{\mathrm{p}}^{2}$. The matrices in Eqs. (17) and (18) are given as

$$
\left.\begin{array}{c}
\mathbf{R}_{0}^{\mathrm{p}, \mathrm{c}}=\mathbf{K}_{0}^{\mathrm{p}, \mathrm{c}}-\mathbf{K}_{2}^{\mathrm{p}, \mathrm{c}}-\gamma_{\mathrm{p}, \mathrm{c}}^{2} \mathbf{S}_{0}^{\mathrm{p}, \mathrm{c}}+\mathbf{S}_{2}^{\mathrm{p}, \mathrm{c}} \\
\mathbf{R}_{1}^{\mathrm{p}}=-i k_{\mathrm{p}}\left\{\left[\frac{a_{\mathrm{p}}^{2}}{c_{\mathrm{p}}^{2}}-1\right]\left(\mathbf{S}_{1}^{\mathrm{p}}+\mathbf{K}_{1}^{\mathrm{p}}\right)+\left.1\right|_{r=a_{1}}-\left.1\right|_{r=b_{1}}\right\} \\
\mathbf{R}_{1}^{\mathrm{c}}=-i k_{\mathrm{p}}\left\{\left[\frac{a_{c}^{2}}{c_{c}^{2}}-1\right]\left(\mathbf{S}_{1}^{\mathrm{c}}+\mathbf{K}_{1}^{\mathrm{c}}\right)+\left.1\right|_{r=b_{1}}-\left.1\right|_{r=b_{2}}\right\} \\
\mathbf{Z}_{0}^{\mathrm{p}, \mathrm{c}}=\mathbf{K}_{3}^{\mathrm{p}, \mathrm{c}}-\mathbf{K}_{2}^{\mathrm{p}, \mathrm{c}}-k_{\mathrm{p}, \mathrm{c}}^{2} \mathbf{S}_{0}^{\mathrm{p}, \mathrm{c}} \\
\mathbf{Z}_{1}^{\mathrm{p}}=-i k_{\mathrm{p}}\left\{\left[1-\frac{c_{\mathrm{p}}^{2}}{a_{\mathrm{p}}^{2}}\right] \mathbf{K}_{1}^{\mathrm{p}}-\left.\left[\frac{2 c_{\mathrm{p}}^{2}}{a_{\mathrm{p}}^{2}}-1\right]\right|_{r=a_{1}}+\left.\left[\frac{c_{\mathrm{p}, \mathrm{c}}^{2}}{a_{\mathrm{p}, \mathrm{c}}^{\mathrm{p}, \mathrm{c}}}+1\right]\right|_{r=b_{1}}\right.
\end{array}\right\}
$$


Matrices $\mathbf{K}$ and $\mathbf{S}$ are defined in Appendix 1. In the matrices above it is assumed that an identical finite element discretisation is used for displacements $u(r)$ and $w(r)$, so that $\mathbf{N}=\mathbf{M}$. Finally, Eqs. (15) and (16) may be grouped together to give

$$
\left[\begin{array}{cccc}
\mathbf{0} & \mathbf{0} & \mathbf{I} & \mathbf{0} \\
\mathbf{0} & \mathbf{0} & \mathbf{0} & \mathbf{I} \\
-\mathbf{R}_{2}^{-1} \mathbf{R}_{0} & \mathbf{0} & \mathbf{0} & -\mathbf{R}_{2}^{-1} \mathbf{Z}_{1} \\
\mathbf{0} & -\mathbf{Z}_{2}^{-1} \mathbf{Z}_{0} & -\mathbf{Z}_{2}^{-1} \mathbf{Z}_{1} & \mathbf{0}
\end{array}\right]\left\{\begin{array}{c}
\mathbf{u} \\
\mathbf{w} \\
\lambda \mathbf{u} \\
\lambda \mathbf{w}
\end{array}\right\}=\lambda\left\{\begin{array}{c}
\mathbf{u} \\
\mathbf{w} \\
\lambda \mathbf{u} \\
\lambda \mathbf{w}
\end{array}\right\}
$$

where I is an identity matrix. Equation (27) is solved using a standard eigenvalue solver (in this case the "eig" function in MATLAB). Equation (27) has order $4 p_{2}$, and on solution of this equation one obtains $2 p_{2}$ unique (complex) eigenmodes for $\lambda$, as well as $2 p_{2}$ solutions for $-\lambda$. These solutions are used in the sections that follow, after first sorting the imaginary components of $\lambda$ into ascending order. It is noted here that the analysis in this section delivers a version of the SAFE method but for longitudinal modes only, and so it is not necessary to sort these modes from other types of modes such as torsional or flexural modes, which is a common problem when using commercial software such as, for example, COMSOL [5] or ABAQUS [10]. The analysis presented in this section is for a coated pipe; however, in the following section eigenvalues and eigenvectors for an uncoated pipe are also required and one may address this problem simply by setting $\sigma=$ $\beta=0$ and putting in the appropriate limits for the integrals in Appendix 1, see Eqs. (A1) to (A8).

\subsection{Mode Matching}

Mode matching is an efficient technique for studying scattering from uniform discontinuities and this approach is used here to analyse an axisymmetric uniform notch of length $L_{N}$ and radius $a_{2}$, which is located within the coated region of the pipe, see Fig. 1. In Fig. 1, region 1 denotes an uncoated region within which an incident wave is generated, as this best replicates actual practice;

the uniform notch of length $L_{N}$ is then located within a (semi-infinite) region of coated pipe where 
the length of the coating section before the notch is $L_{\mathrm{C}}$. The displacement in region $q$ (where $q=1,2,3$, or 4 ) of the pipe is written as a sum over the pipe eigenmodes, to give

$$
\mathbf{Y}_{q}^{\prime}(r, z)=\sum_{n=0}^{n_{q}} A_{q}^{n} \mathbf{Y}_{q+}^{n}(r) \mathrm{e}^{-i k_{\mathrm{p}} \lambda_{q}^{n} z_{q}}+\sum_{n=0}^{n_{q}} B_{q}^{n} \mathbf{Y}_{q-}^{n}(r) \mathrm{e}^{i k_{\mathrm{p}} \lambda_{q}^{n} z_{q}}
$$

where $A_{q}$ and $B_{q}$ are the modal amplitudes in region $q$, and $z_{1}=z_{2}$. Here, the modal sums are truncated at $n_{q}$. The eigenvalues $\lambda_{q}$ and associated eigenvectors in each region are found using the method described in section 2.1. Note that in Eq. (28) it is necessary to distinguish between the incident $(+)$ and reflected (-) eigenvectors, which are different for longitudinal modes. In addition $\mathbf{Y}_{q}^{\prime}(r, z)=\left[\begin{array}{ll}u_{q}^{\prime}(r, z) & w_{q}^{\prime}(r, z)\end{array}\right]^{\mathrm{T}}$, so that

$$
\mathbf{Y}_{q \pm}^{n}(r)=\left[\mp u_{q}^{n}(r) \quad w_{q}^{n}(r)\right]^{\mathrm{T}}
$$

where $u(r)$ and $w(r)$ are the eigenvectors associated with the $-\lambda$ solutions to Eq. (27) (the choice of which solution to take here is, of course, arbitrary). The (normalised) shear stress may be expanded in the same way, to give

$$
\widetilde{\mathbf{T}}_{q}^{\prime}(r, z)=\tilde{\sigma}\left[\sum_{n=0}^{n_{q}} A_{q}^{n} \widetilde{\mathbf{T}}_{q+}^{n}(r) \mathrm{e}^{-i k_{\mathrm{p}} \lambda_{q}^{n} z_{q}}+\sum_{n=0}^{n_{q}} B_{q}^{n} \widetilde{\mathbf{T}}_{q-}^{n}(r) \mathrm{e}^{i k_{\mathrm{p}} \lambda_{q}^{n} z_{q}}\right]
$$

where the shear stress is normalised by $\rho_{\mathrm{p}} a_{\mathrm{p}}^{2}$, so that $\widetilde{\mathbf{T}}=\mathbf{T} / \rho_{\mathrm{p}} a_{\mathrm{p}}^{2}$, and so on. Here, $\tilde{\sigma}=1$ in an uncoated region, and $\tilde{\sigma}=\sigma$ in a coated region, with $\widetilde{\mathbf{T}}_{q}^{\prime}(r, z)=\left[\begin{array}{ll}\widetilde{T}_{r z q}^{\prime}(r, z) & \tilde{T}_{z z q}^{\prime}(r, z)\end{array}\right]^{\mathrm{T}}$, and

$$
\widetilde{\mathbf{T}}_{q \pm}^{n}(r)=\left[\tilde{T}_{r z q}^{n}(r) \quad \mp \tilde{T}_{z z q}^{n}(r)\right]^{\mathrm{T}}
$$

In addition, for region $q$, 


$$
\tilde{T}_{r z_{\mathrm{p}, \mathrm{c}}}^{n}(r)=\frac{c_{\mathrm{p}, \mathrm{c}}^{2}}{a_{\mathrm{p}, \mathrm{c}}^{2}}\left[i k_{\mathrm{p}} \lambda^{n} u_{\mathrm{p}, \mathrm{c}}^{n}(r)+\frac{\partial w_{\mathrm{p}, \mathrm{c}}^{n}(r)}{\partial r}\right]
$$

and

$$
\tilde{T}_{z z_{\mathrm{p}, \mathrm{c}}}^{n}(r)=i k_{\mathrm{p}} \lambda^{n} w_{\mathrm{p}, \mathrm{c}}^{n}(r)+\left[\frac{2 c_{\mathrm{p}, \mathrm{c}}^{2}}{a_{\mathrm{p}, \mathrm{c}}^{2}}-1\right]\left(\frac{\partial u_{\mathrm{p}, \mathrm{c}}^{n}(r)}{\partial r}+\frac{u_{\mathrm{p}, \mathrm{c}}^{n}(r)}{r}\right) .
$$

Mode matching proceeds by enforcing axial continuity conditions over planes A, B and C in Fig.

1. The appropriate axial continuity conditions over each plane are (i) continuity of displacement, and (ii) continuity of the shear stresses $\tilde{T}_{r z}$ and $\tilde{T}_{z z}$. In addition, the boundary conditions $\tilde{T}_{r z}=0$ and $\tilde{T}_{z Z}=0$ are enforced over $b_{1}<r<b_{2}$ on plane A, and over $a_{2}<r<b_{2}$ on planes B and C. Following Kirby [1, 20], these axial boundary conditions are enforced by choosing an appropriate weighting function and then integrating over the relevant cross section. For longitudinal modes, the weighting function is chosen so that, where possible, one obtains a bi-orthogonality relation of the type described by, for example, Gregory [24]. Application of these matching conditions using Eqs. (28) and (29) yields six coupled equations, after making use of Eqs. (29) and (31) to (33). Solution of these equations requires a forcing term to be identified and here the model can readily be amended to drive any longitudinal mode in region 1, provided one is operating at a frequency in which the chosen mode is cut on; for example, for $\mathrm{L}(0,1)$ one sets $A_{1}^{0}=1$, with $A_{1}^{n}=0$ for $n>0$, and for $\mathrm{L}(0,2)$ one sets $A_{1}^{1}=1$, with $A_{1}^{0}=0$ and $A_{1}^{n}=0$ for $n>1$ (of course, the same principles apply to any other mode). It is necessary also to apply an axial boundary condition at $z_{4}=\infty$, and here it is assumed that no reflections exist, so that $B_{4}^{n}=0$ for $n \geq 0$. Substituting these axial boundary conditions into the relevant matching conditions and re-arranging so that the forcing terms lie on the right hand side, yields

$$
\begin{aligned}
& B_{1}^{n} \mathrm{I}_{11-}^{m n}-A_{2}^{n} \mathrm{I}_{12+}^{m n}-\tilde{B}_{2}^{n} \mathrm{I}_{12-}^{m n} \mathrm{e}^{-\mathrm{i} k_{\mathrm{p}} \lambda_{2}^{n} L_{\mathrm{c}}}=-A_{1}^{E} \mathrm{I}_{11+}^{m E} \\
& B_{1}^{n} \mathrm{I}_{21-}^{m n}-A_{2}^{n} \mathrm{I}_{22+}^{m n}-\tilde{B}_{2}^{n} \mathrm{I}_{22-}^{m n} \mathrm{e}^{-\mathrm{i} k_{\mathrm{p}} \lambda_{2}^{n} L_{\mathrm{c}}}=-A_{1}^{E} \mathrm{I}_{21+}^{m E}
\end{aligned}
$$




$$
\begin{gathered}
A_{2}^{n} \mathrm{I}_{32+}^{m n} \mathrm{e}^{-\mathrm{i} k_{\mathrm{p}} \lambda_{2}^{n} L_{\mathrm{c}}}+\tilde{B}_{2}^{n} \mathrm{I}_{32-}^{m n}-A_{3}^{n} \mathrm{I}_{33+}^{m n}-\tilde{B}_{3}^{n} \mathrm{I}_{33-}^{m n} \mathrm{e}^{-\mathrm{i} k_{\mathrm{p}} \lambda_{3}^{n} L_{\mathrm{N}}}=0 \\
A_{2}^{n} \mathrm{H}_{22+}^{m n} \mathrm{e}^{-\mathrm{i} k_{\mathrm{p}} \lambda_{2}^{n} L_{\mathrm{c}}}+\tilde{B}_{2}^{n} \mathrm{H}_{22-}^{m n}-A_{3}^{n} \mathrm{H}_{23+}^{m n}-\tilde{B}_{3}^{n} \mathrm{H}_{23-}^{m n} \mathrm{e}^{-\mathrm{i} k_{\mathrm{p}} \lambda_{3}^{n} L_{\mathrm{N}}}=0 \\
A_{3}^{n} \mathrm{H}_{33+}^{m n} \mathrm{e}^{-\mathrm{i} k_{\mathrm{p}} \lambda_{3}^{n} L_{\mathrm{N}}}+\tilde{B}_{3}^{n} \mathrm{H}_{33-}^{m n}-A_{4}^{n} \mathrm{H}_{32+}^{m n}=0 \\
A_{3}^{n} \mathrm{~J}_{23+}^{m n} \mathrm{e}^{-\mathrm{i} k_{\mathrm{p}} \lambda_{3}^{n} L_{\mathrm{N}}}+\tilde{B}_{3}^{n} \mathrm{~J}_{23-}^{m n}-A_{4}^{n} \mathrm{I}_{22+}^{m n}=0 .
\end{gathered}
$$

Here, it is assumed that regions 2 and 4 are identical, with $\widetilde{B}_{2}^{n}=B_{2}^{n} \mathrm{e}^{-\mathrm{i} k_{\mathrm{p}} \lambda_{2}^{n} L_{\mathrm{c}}}$ and $\tilde{B}_{3}^{n}=$ $B_{3}^{n} \mathrm{e}^{-\mathrm{i} k_{\mathrm{p}} \lambda_{3}^{n} L_{\mathrm{N}}}$. Further, for the right hand side of Eqs. (34) and (35), $E=0$ when driving $\mathrm{L}(0,1)$, and $E=1$ if driving $\mathrm{L}(0,2)$, and so on. The integrals in Eqs, (34) to (39) are given in Appendix 2 , and these equations are then solved simultaneously for the $\left(n_{t}=n_{1}+3 n_{2}+2 n_{3}\right)$ unknown modal amplitudes.

\subsection{Hybrid numerical method}

For non-uniform discontinuities it is necessary to return to a full finite element discretisation of the defect in order to accommodate non-uniform (but axisymmetric) geometries, see Fig. 2. Accordingly, a hybrid method is adopted here, which follows the method described by Kirby [1, 20, 25], and so the displacements in region 3 of Fig. 2 are discretised to give

$$
u_{3}^{\prime}(r, z)=\sum_{j=1}^{m_{3}} R_{j}(r, z) u_{3 j}
$$

and

$$
w_{3}^{\prime}(r, z)=\sum_{j=1}^{m_{3}} Z_{j}(r, z) w_{3 j}
$$

where $R_{j}$ and $Z_{j}$ are a global trial (or shape) functions for the (two dimensional) finite element mesh; and in vector form $u_{3}^{\prime}(r, z)=\mathbf{R} \mathbf{u}_{3}$ and $w_{3}^{\prime}(r, z)=\mathbf{Z} \mathbf{w}_{3}$. A weak Galerkin formulation is applied and follows the method described in the companion paper on torsional modes [1]. 
Accordingly, the analysis proceeds by enforcing appropriate boundary conditions over the surface of $\Omega_{3 \mathrm{p}, \mathrm{c}}$. Over the interface between the substrate and the coating the shear stress $T_{r r}^{\prime} \cdot \mathbf{n}_{r}$ is continuous for the radial displacment, and $T_{r Z}^{\prime} \cdot \mathbf{n}_{r}$ is continuous for the axial displacement, where $\mathbf{n}_{r}$ and $\mathbf{n}_{z}$ are unit normals pointing outwards from surface $\Gamma_{3 p, c}$ in the $r$ and $z$ direction, respectively. Over the surfaces $\Gamma_{3 \mathrm{p}}$ and $\Gamma_{3 \mathrm{c}}$ that do not lie on this interface, $T_{r r}^{\prime} \cdot \mathbf{n}_{r}=T_{r z}^{\prime} \cdot \mathbf{n}_{r}=$ $T_{r z}^{\prime} \cdot \mathbf{n}_{z}=T_{z z}^{\prime} \cdot \mathbf{n}_{z}=0$. Over $\Gamma_{\mathrm{X}}$ and $\Gamma_{\mathrm{Y}}, T_{r z}^{\prime} \cdot \mathbf{n}_{z}$ and $T_{z z}^{\prime} \cdot \mathbf{n}_{z}$ are continuous for radial and axial displacements, respectively. Finally, the displacements in the axial and radial direction are continuous over $\Gamma_{\mathrm{X}}$ and $\Gamma_{\mathrm{Y}}$, as well as the interface between the substrate and the coating (see Fig. 2). Here, the shear stresses are given in general as

$$
\begin{gathered}
\frac{T_{r r_{\mathrm{p}, \mathrm{c}}}^{\prime}}{\rho_{\mathrm{p}, \mathrm{c}} a_{\mathrm{p}, \mathrm{c}}^{2}}=\frac{\partial u_{\mathrm{p}, \mathrm{c}}^{\prime}}{\partial r}-\left[\frac{2 c_{\mathrm{p}, \mathrm{c}}^{2}}{a_{\mathrm{p}, \mathrm{c}}^{2}}-1\right]\left(\frac{u_{\mathrm{p}, \mathrm{c}}^{\prime}}{r}+\frac{\partial w_{\mathrm{p}, \mathrm{c}}^{\prime}}{\partial z}\right), \\
\frac{T_{r z_{\mathrm{p}, \mathrm{c}}}^{\prime}}{\rho_{\mathrm{p}, \mathrm{c}} a_{\mathrm{p}, \mathrm{c}}^{2}}=\frac{\partial u_{\mathrm{p}, \mathrm{c}}^{\prime}}{\partial z}+\frac{\partial w_{\mathrm{p}, \mathrm{c}}^{\prime}}{\partial r}
\end{gathered}
$$

and

$$
\frac{T_{z z_{\mathrm{p}, \mathrm{c}}}^{\prime}}{\rho_{\mathrm{p}, \mathrm{c}} a_{\mathrm{p}, \mathrm{c}}^{2}}=\frac{\partial w_{\mathrm{p}, \mathrm{c}}^{\prime}}{\partial z}-\left[\frac{2 c_{\mathrm{p}, \mathrm{c}}^{2}}{a_{\mathrm{p}, \mathrm{c}}^{2}}-1\right]\left(\frac{u_{\mathrm{p}, \mathrm{c}}^{\prime}}{r}+\frac{\partial u_{\mathrm{p}, \mathrm{c}}^{\prime}}{\partial r}\right) .
$$

These expressions for shear stress are first re-arranged and then substituted into the boundary integrals that arise from the weak Galerkin formulation. This then allows the equations for the substrate and the coating region to be joined together and, after some rearranging, this gives 


$$
\begin{aligned}
& \left\{\int_{\Omega_{3 \mathrm{p}}}\left[\frac{\partial \mathbf{R}^{\mathrm{T}}}{\partial r} \frac{\partial \mathbf{R}}{\partial r}+\frac{c_{\mathrm{p}}^{2}}{a_{\mathrm{p}}^{2}} \frac{\partial \mathbf{R}^{\mathrm{T}}}{\partial z} \frac{\partial \mathbf{R}}{\partial z}-\frac{\mathbf{R}^{\mathrm{T}}}{r} \frac{\partial \mathbf{R}}{\partial z}+\frac{\mathbf{R}^{\mathrm{T}} \mathbf{R}}{r^{2}}-\gamma_{\mathrm{p}}^{2} \mathbf{R}^{\mathrm{T}} \mathbf{R}\right] d \Omega_{3 \mathrm{p}}\right\}\left\{\mathbf{u}_{3 \mathrm{p}}\right\} \\
& +\sigma\left\{\int_{\Omega_{3 c}}\left[\frac{\partial \mathbf{R}^{\mathrm{T}}}{\partial r} \frac{\partial \mathbf{R}}{\partial r}+\frac{c_{\mathrm{c}}^{2}}{a_{\mathrm{c}}^{2}} \frac{\partial \mathbf{R}^{\mathrm{T}}}{\partial z} \frac{\partial \mathbf{R}}{\partial z}-\frac{\mathbf{R}^{\mathrm{T}}}{r} \frac{\partial \mathbf{R}}{\partial z}+\frac{\mathbf{R}^{\mathrm{T}} \mathbf{R}}{r^{2}}-\gamma_{\mathrm{c}}^{2} \mathbf{R}^{\mathrm{T}} \mathbf{R}\right] d \Omega_{3 \mathrm{c}}\right\}\left\{\mathbf{u}_{3 \mathrm{c}}\right\} \\
& +\left\{\int_{\Omega_{3 \mathrm{p}}}\left[\frac{\partial \mathbf{Z}^{\mathrm{T}}}{\partial r} \frac{\partial \mathbf{Z}}{\partial z}-\frac{c_{\mathrm{p}}^{2}}{a_{\mathrm{p}}^{2}} \frac{\partial \mathbf{Z}^{\mathrm{T}}}{\partial z} \frac{\partial \mathbf{Z}}{\partial r}\right] d \Omega_{3 \mathrm{p}}\right\}\left\{\mathbf{w}_{3 \mathrm{p}}\right\}+\sigma\left\{\int_{\Omega_{3 \mathrm{c}}}\left[\frac{\partial \mathbf{Z}^{\mathrm{T}}}{\partial r} \frac{\partial \mathbf{Z}}{\partial z}-\frac{c_{\mathrm{c}}^{2}}{a_{\mathrm{c}}^{2}} \frac{\partial \mathbf{Z}^{\mathrm{T}}}{\partial z} \frac{\partial \mathbf{Z}}{\partial r}\right] d \Omega_{3 \mathrm{c}}\right\}\left\{\mathbf{w}_{3 \mathrm{c}}\right\} \\
& =2 \frac{c_{\mathrm{p}}^{2}}{a_{\mathrm{p}}^{2}}\left\{\int_{\Gamma_{3 \mathrm{p}}}\left[\mathbf{R}^{\mathrm{T}} \frac{\partial \mathbf{Z}}{\partial r} \cdot \mathbf{n}_{z}-\mathbf{R}^{\mathrm{T}} \frac{\partial \mathbf{Z}}{\partial z} \cdot \mathbf{n}_{r}\right] d \Gamma_{3 \mathrm{p}}\right\}\left\{\mathbf{w}_{3 \mathrm{p}}\right\} \\
& +2 \sigma \frac{c_{\mathrm{c}}^{2}}{a_{\mathrm{c}}^{2}}\left\{\int_{\Gamma_{3 \mathrm{c}}}\left[\mathbf{R}^{\mathrm{T}} \frac{\partial \mathbf{Z}}{\partial r} \cdot \mathbf{n}_{z}-\mathbf{R}^{\mathrm{T}} \frac{\partial \mathbf{Z}}{\partial z} \cdot \mathbf{n}_{r}\right] d \Gamma_{3 \mathrm{c}}\right\}\left\{\mathbf{w}_{3 \mathrm{c}}\right\} \\
& -\left[2 \frac{c_{\mathrm{p}}^{2}}{a_{\mathrm{p}}^{2}}-1\right]\left\{\int_{\Gamma_{3 \mathrm{p}}} \frac{\mathbf{R}^{\mathrm{T}} \mathbf{R}}{r} \cdot \mathbf{n}_{r} d \Gamma_{3 \mathrm{p}}\right\}\left\{\mathbf{u}_{3 \mathrm{p}}\right\}-\sigma\left[2 \frac{c_{\mathrm{c}}^{2}}{a_{\mathrm{c}}^{2}}-1\right]\left\{\int_{\Gamma_{3 \mathrm{c}}} \frac{\mathbf{R}^{\mathrm{T}} \mathbf{R}}{r} \cdot \mathbf{n}_{r} d \Gamma_{3 \mathrm{c}}\right\}\left\{\mathbf{u}_{3 \mathrm{c}}\right\} \\
& +\int_{\Gamma_{\mathrm{Xp}}} \mathbf{R}^{\mathrm{T}} \frac{T_{r z_{\mathrm{p}}}^{\prime}}{\rho_{\mathrm{p}} a_{\mathrm{p}}^{2}} d \Gamma_{\mathrm{Xp}}+\int_{\Gamma_{\mathrm{Xc}}} \mathbf{R}^{\mathrm{T}} \frac{T_{r z_{\mathrm{c}}}^{\prime}}{\rho_{\mathrm{c}} a_{\mathrm{c}}^{2}} d \Gamma_{\mathrm{Xc}}-\int_{\Gamma_{\mathrm{Yp}}} \mathbf{R}^{\mathrm{T}} \frac{T_{r z_{\mathrm{p}}}^{\prime}}{\rho_{\mathrm{p}} a_{\mathrm{p}}^{2}} d \Gamma_{\mathrm{Yp}}-\int_{\Gamma_{\mathrm{Yc}}} \mathbf{R}^{\mathrm{T}} \frac{T_{r z_{\mathrm{c}}}^{\prime}}{\rho_{\mathrm{c}} a_{\mathrm{c}}^{2}} d \Gamma_{\mathrm{Yc}} .
\end{aligned}
$$


and

$$
\begin{aligned}
& \left\{\int_{\Omega_{3 \mathrm{p}}}\left[\frac{c_{\mathrm{p}}^{2}}{a_{\mathrm{p}}^{2}} \frac{\partial \mathbf{Z}^{\mathrm{T}}}{\partial r} \frac{\partial \mathbf{Z}}{\partial r}+\frac{\partial \mathbf{Z}^{\mathrm{T}}}{\partial z} \frac{\partial \mathbf{Z}}{\partial z}-\frac{c_{\mathrm{p}}^{2}}{a_{\mathrm{p}}^{2}} \frac{\mathbf{Z}^{\mathrm{T}}}{r} \frac{\partial \mathbf{Z}}{\partial r}-\gamma_{\mathrm{p}}^{2} \mathbf{Z}^{\mathrm{T}} \mathbf{Z}\right] d \Omega_{3 \mathrm{p}}\right\}\left\{\mathbf{w}_{3 \mathrm{p}}\right\} \\
& +\sigma\left\{\int_{\Omega_{3 \mathrm{c}}}\left[\frac{c_{\mathrm{c}}^{2}}{a_{\mathrm{c}}^{2}} \frac{\partial \mathbf{Z}^{\mathrm{T}}}{\partial r} \frac{\partial \mathbf{Z}}{\partial r}+\frac{\partial \mathbf{Z}^{\mathrm{T}}}{\partial z} \frac{\partial \mathbf{Z}}{\partial z}-\frac{c_{\mathrm{c}}^{2}}{a_{\mathrm{c}}^{2}} \frac{\mathbf{Z}^{\mathrm{T}}}{r} \frac{\partial \mathbf{Z}}{\partial r}-\gamma_{\mathrm{c}}^{2} \mathbf{Z}^{\mathrm{T}} \mathbf{Z}\right] d \Omega_{3 \mathrm{c}}\right\}\left\{\mathbf{w}_{3 \mathrm{c}}\right\} \\
& +\left\{\int_{\Omega_{3 \mathrm{p}}}\left[\frac{\partial \mathbf{R}^{\mathrm{T}}}{\partial z} \frac{\partial \mathbf{R}}{\partial r}-\frac{c_{\mathrm{p}}^{2}}{a_{\mathrm{p}}^{2}} \frac{\partial \mathbf{R}^{\mathrm{T}}}{\partial r} \frac{\partial \mathbf{R}}{\partial z}+\left(\frac{c_{\mathrm{p}}^{2}}{a_{\mathrm{p}}^{2}}-1\right) \frac{\mathbf{R}^{\mathrm{T}}}{r} \frac{\partial \mathbf{R}}{\partial z}\right] d \Omega_{3 \mathrm{p}}\right\}\left\{\mathbf{u}_{3 \mathrm{p}}\right\} \\
& +\sigma\left\{\int_{\Omega_{3 c}}\left[\frac{\partial \mathbf{R}^{\mathrm{T}}}{\partial z} \frac{\partial \mathbf{R}}{\partial r}-\frac{c_{\mathrm{c}}^{2}}{a_{\mathrm{c}}^{2}} \frac{\partial \mathbf{R}^{\mathrm{T}}}{\partial r} \frac{\partial \mathbf{R}}{\partial z}+\left(\frac{c_{\mathrm{c}}^{2}}{a_{\mathrm{c}}^{2}}-1\right) \frac{\mathbf{R}^{\mathrm{T}}}{r} \frac{\partial \mathbf{R}}{\partial z}\right] d \Omega_{3 \mathrm{c}}\right\}\left\{\mathbf{u}_{3 \mathrm{c}}\right\} \\
& =2 \frac{c_{\mathrm{p}}^{2}}{a_{\mathrm{p}}^{2}}\left\{\int_{\Gamma_{3 \mathrm{p}}}\left[\mathbf{Z}^{\mathrm{T}} \frac{\partial \mathbf{R}}{\partial r} \cdot \mathbf{n}_{z}-\mathbf{R}^{\mathrm{T}} \frac{\partial \mathbf{R}}{\partial z} \cdot \mathbf{n}_{r}\right] d \Gamma_{3 \mathrm{p}}\right\}\left\{\mathbf{u}_{3 \mathrm{p}}\right\} \\
& +2 \sigma \frac{c_{\mathrm{c}}^{2}}{a_{\mathrm{c}}^{2}}\left\{\int_{\Gamma_{3 \mathrm{c}}}\left[\mathbf{Z}^{\mathrm{T}} \frac{\partial \mathbf{R}}{\partial r} \cdot \mathbf{n}_{z}-\mathbf{R}^{\mathrm{T}} \frac{\partial \mathbf{R}}{\partial z} \cdot \mathbf{n}_{r}\right] d \Gamma_{3 \mathrm{c}}\right\}\left\{\mathbf{u}_{3 \mathrm{c}}\right\} \\
& +\left[2 \frac{c_{\mathrm{p}}^{2}}{a_{\mathrm{p}}^{2}}-1\right]\left\{\int_{\Gamma_{3 \mathrm{p}}} \frac{\mathbf{Z}^{\mathrm{T}} \mathbf{R}}{r} \cdot \mathbf{n}_{z} d \Gamma_{3 \mathrm{p}}\right\}\left\{\mathbf{u}_{3 \mathrm{p}}\right\}+\sigma\left[2 \frac{c_{\mathrm{c}}^{2}}{a_{\mathrm{c}}^{2}}-1\right]\left\{\int_{\Gamma_{3 \mathrm{c}}} \frac{\mathbf{Z}^{\mathrm{T}} \mathbf{R}}{r} \cdot \mathbf{n}_{z} d \Gamma_{3 \mathrm{c}}\right\}\left\{\mathbf{u}_{3 \mathrm{c}}\right\} \\
& -\int_{\Gamma_{\mathrm{Xp}}} \mathbf{Z}^{\mathrm{T}} \frac{T_{z z_{\mathrm{p}}}^{\prime}}{\rho_{\mathrm{p}} a_{\mathrm{p}}^{2}} d \Gamma_{\mathrm{Xp}}-\int_{\Gamma_{\mathrm{Xc}}} \mathbf{Z}^{\mathrm{T}} \frac{T_{z z_{\mathrm{c}}}^{\prime}}{\rho_{\mathrm{c}} a_{\mathrm{c}}^{2}} d \Gamma_{\mathrm{Xc}}+\int_{\Gamma_{\mathrm{Yp}}} \mathbf{R}^{\mathrm{T}} \frac{T_{z z_{\mathrm{p}}}^{\prime}}{\rho_{\mathrm{p}} a_{\mathrm{p}}^{2}} d \Gamma_{\mathrm{Yp}}+\int_{\Gamma_{\mathrm{Yc}}} \mathbf{R}^{\mathrm{T}} \frac{T_{z z_{\mathrm{c}}}^{\prime}}{\rho_{\mathrm{c}} a_{\mathrm{c}}^{2}} d \Gamma_{\mathrm{Yc}} .
\end{aligned}
$$

Here, $\mathbf{u}_{3}=\left[\begin{array}{ll}\mathbf{u}_{3 p} & \mathbf{u}_{3 c}\end{array}\right]^{T}$ and $\mathbf{w}_{3}=\left[\begin{array}{ll}\mathbf{w}_{3 p} & \mathbf{w}_{3 c}\end{array}\right]^{\mathrm{T}}$, and it has been assumed that $\mathbf{R}=\mathbf{Z}$ when grouping together some of the terms on the right hand side of Eqs. (42) and (43). These two equations have been written out in full here as this best illustrates how the boundary conditions are implemented. It is evident that after application of the boundary conditions over all surfaces, the shear stress $\left(T^{\prime}\right)$ now only appears over the inlet and outlet planes of region 3 (planes $\mathrm{X}$ and $\mathrm{Y}$ ), which is where the hybrid method is used to join the finite element discretisation to the modal expansions in regions 2 and 4. Accordingly, over boundaries $\Gamma_{\mathrm{X}}$ and $\Gamma_{\mathrm{Y}}$, continuity of the shear stresses $T_{r z_{\mathrm{p}}}^{\prime}$ and $T_{z z_{\mathrm{p}}}^{\prime}$ is enforced by equating the $T^{\prime}$ terms on the right hand side of Eqs. (42) and 
(43) with a modal expansion for the shear stress given by Eq. (30). Following this, Eqs. (42) and (43) are written in vector form to give

$$
\mathbf{K}_{1 r} \mathbf{u}_{3}+\mathbf{K}_{1 z} \mathbf{W}_{3}+\mathbf{D} \mathbf{Q}_{1}^{\mathrm{T}} \widetilde{\mathbf{A}}_{2}+\mathbf{Q}_{1}^{\mathrm{T}} \mathbf{B}_{2}+\mathbf{R}_{1}^{\mathrm{T}} \mathbf{A}_{4}=0
$$

and

$$
\mathbf{K}_{2 z} \mathbf{w}_{3}+\mathbf{K}_{2 r} \mathbf{u}_{3}-\mathbf{D} \mathbf{Q}_{2}^{\mathrm{T}} \widetilde{\mathbf{A}}_{2}+\mathbf{Q}_{2}^{\mathrm{T}} \mathbf{B}_{2}+\mathbf{R}_{2}^{\mathrm{T}} \mathbf{A}_{4}=0
$$

Here, it is assumed that no reflections are present in region 4 , so that $B_{4}^{m}=0$ for all $m$, and the normalised values for the shear stress are given by Eqs. (32) and (33). In addition $\mathbf{D}$ is a diagonal matrix with each diagonal element given by $\mathrm{e}^{-i k_{\mathrm{p}} \lambda_{2}^{n} L_{2}}$ for $n=0,1, \ldots n_{2}$, so that $\mathbf{D} \widetilde{\mathbf{A}}_{2}=\mathbf{A}_{2}$. Here, $L_{2}$ is the distance between planes $\mathrm{A}$ and $\mathrm{X}$ in Fig. 2. Further, $\mathbf{A}_{q}$ and $\mathbf{B}_{q}$ are column vectors of length $n_{q}$, which hold the modal amplitudes for regions 1,2 and 4 .

Continuity of displacement is matched separately using mode matching, which gives

$$
\mathbf{D} J_{22}^{m n} \widetilde{\mathbf{A}}_{2}+J_{22-}^{m n} \mathbf{B}_{2}+\mathbf{Q}_{1} \mathbf{u}_{3 \mathrm{X}}+\mathbf{Q}_{2} \mathbf{w}_{3 \mathrm{X}}=0
$$

and

$$
-J_{22-}^{m n} \mathbf{B}_{4}+\mathbf{R}_{1}^{\mathrm{T}} \mathbf{u}_{3 \mathrm{Y}}+\mathbf{R}_{2}^{\mathrm{T}} \mathbf{w}_{3 \mathrm{Y}}=0
$$

Matrices $J_{22 \pm}^{m n}$ are defined in Appendix 2, and vectors $\mathbf{u}_{3 \mathrm{X}}$ and $\mathbf{w}_{3 \mathrm{X}}$ hold the values of the finite element solution in region 3 at the nodal locations on $\Gamma_{\mathrm{X}}$, with $\mathbf{u}_{3 \mathrm{Y}}$ and $\mathbf{w}_{3 \mathrm{Y}}$ holding these values for $\Gamma_{\mathrm{Y}}$.

Finally, mode matching is used to accommodate the discontinuity between regions 1 and 2 using the same method as that described in the previous section, see Eqs. (34) and (35). To combine Eqs. (34), (35) and (44) to (47), matrices $\mathbf{K}_{1 r}, \mathbf{K}_{1 z} \mathbf{K}_{2 r}, \mathbf{K}_{2 z}$ are first decomposed into matrices $\mathbf{G}^{1 r}$, $\mathbf{G}^{1 z}, \mathbf{G}^{2 r}$ and $\mathbf{G}^{2 z}$, respectively. This allows those elements that lie on planes $\mathrm{X}$ and $\mathrm{Y}$ to be 
separated for the application of the matching conditions, and the final system of equations is then written as

$$
\left[\begin{array}{cccccccccc}
I_{11-}^{m n} & -I_{12+}^{m n} & -\mathbf{D} I_{12-}^{m n} & \mathbf{0} & \mathbf{0} & \mathbf{0} & \mathbf{0} & \mathbf{0} & \mathbf{0} & \mathbf{0} \\
-I_{21-}^{m n} & I_{22+}^{m n} & -\mathbf{D} I_{22-}^{m n} & \mathbf{0} & \mathbf{0} & \mathbf{0} & \mathbf{0} & \mathbf{0} & \mathbf{0} & \mathbf{0} \\
\mathbf{0} & \mathbf{D} J_{22+}^{m n} & J_{22-}^{m n} & \mathbf{Q}_{1} & \mathbf{0} & \mathbf{0} & \mathbf{Q}_{2} & \mathbf{0} & \mathbf{0} & \mathbf{0} \\
\mathbf{0} & \mathbf{D} \mathbf{Q}_{1}^{\mathrm{T}} & \mathbf{Q}_{1}^{\mathrm{T}} & \mathbf{G}_{\mathrm{XX}}^{1 r} & \mathbf{G}_{\mathrm{Xe}}^{1 r} & \mathbf{G}_{\mathrm{XY}}^{1 r} & \mathbf{G}_{\mathrm{XX}}^{1 z} & \mathbf{G}_{\mathrm{Xe}}^{1 z} & \mathbf{G}_{\mathrm{XY}}^{1 z} & \mathbf{0} \\
\mathbf{0} & \mathbf{0} & \mathbf{0} & \mathbf{G}_{\mathrm{eX}}^{1 r} & \mathbf{G}_{\mathrm{ee}}^{1 r} & \mathbf{G}_{\mathrm{eY}}^{1 r} & \mathbf{G}_{\mathrm{eX}}^{1 z} & \mathbf{G}_{\mathrm{ee}}^{1 z} & \mathbf{G}_{\mathrm{eY}}^{1 z} & \mathbf{0} \\
\mathbf{0} & \mathbf{0} & \mathbf{0} & \mathbf{G}_{\mathrm{YX}}^{1 r} & \mathbf{G}_{\mathrm{Ye}}^{1 r} & \mathbf{G}_{\mathrm{YY}}^{1 r} & \mathbf{G}_{\mathrm{YX}}^{1 z} & \mathbf{G}_{\mathrm{Ye}}^{1 z} & \mathbf{G}_{\mathrm{YY}}^{1 z} & \mathbf{R}_{1} \\
\mathbf{0} & -\mathbf{D} \mathbf{Q}_{2}^{\mathrm{T}} & \mathbf{Q}_{2}^{\mathrm{T}} & \mathbf{G}_{\mathrm{XX}}^{2 r} & \mathbf{G}_{\mathrm{Xe}}^{2 r} & \mathbf{G}_{\mathrm{XY}}^{2 r} & \mathbf{G}_{\mathrm{XX}}^{2 z} & \mathbf{G}_{\mathrm{Xe}}^{2 z} & \mathbf{G}_{\mathrm{XY}}^{2 z} & \mathbf{0} \\
\mathbf{0} & \mathbf{0} & \mathbf{0} & \mathbf{G}_{\mathrm{eX}}^{2 r} & \mathbf{G}_{\mathrm{ee}}^{2 r} & \mathbf{G}_{\mathrm{eY}}^{2 r} & \mathbf{G}_{\mathrm{eX}}^{2 z} & \mathbf{G}_{\mathrm{ee}}^{2 z} & \mathbf{G}_{\mathrm{eY}}^{2 z} & \mathbf{0} \\
\mathbf{0} & \mathbf{0} & \mathbf{0} & \mathbf{G}_{\mathrm{YX}}^{2 r} & \mathbf{G}_{\mathrm{Ye}}^{2 r} & \mathbf{G}_{\mathrm{YY}}^{2 r} & \mathbf{G}_{\mathrm{YX}}^{2 z} & \mathbf{G}_{\mathrm{Ye}}^{2 z} & \mathbf{G}_{\mathrm{YY}}^{2 z} & \mathbf{R}_{2} \\
\mathbf{0} & \mathbf{0} & \mathbf{0} & \mathbf{0} & \mathbf{0} & \mathbf{R}_{1}^{\mathrm{T}} & \mathbf{0} & \mathbf{0} & \mathbf{R}_{2}^{\mathrm{T}} & -J_{22}^{m n}
\end{array}\right]\left[\begin{array}{c}
\mathbf{B}_{1} \\
\widetilde{\mathbf{A}}_{2} \\
\mathbf{B}_{2} \\
\mathbf{u}_{3 \mathrm{X}} \\
\mathbf{u}_{3 \mathrm{e}} \\
\mathbf{u}_{3 \mathrm{Y}} \\
\mathbf{w}_{3 \mathrm{X}} \\
\mathbf{w}_{3 \mathrm{e}} \\
\mathbf{w}_{3 \mathrm{Y}} \\
\mathbf{A}_{4}
\end{array}\right]=\left[\begin{array}{c}
-A_{1}^{E} I_{11+}^{m E} \\
-A_{1}^{E} I_{21+}^{m E} \\
0 \\
\mathbf{0} \\
\mathbf{0} \\
\mathbf{0} \\
\mathbf{0} \\
\mathbf{0} \\
\mathbf{0} \\
0
\end{array}\right]
$$

Here, matrix $G_{m n}$ has order $p_{m} \times p_{n}$, where $p_{2}$ and $p_{4}$ denote the number of nodes on $\Gamma_{\mathrm{X}}$ and $\Gamma_{\mathrm{Y}}$, respectively (where $n_{2} \leq p_{2}$, and $n_{4} \leq p_{2}$ ); $p_{3}$ is the number of nodes in region 3 , and $s_{e}$ is the number of nodes that lie in region 3 , but do not lie on $\Gamma_{\mathrm{X}}$ and $\Gamma_{\mathrm{Y}}$ (so that $p_{e}=p_{3}-p_{2}-p_{4}$ ). The values for displacements at those nodes in region 3 that do not lie on $\Gamma_{\mathrm{X}}$ and $\Gamma_{\mathrm{Y}}$, are held in matrices $\mathbf{u}_{3 \mathrm{e}}$ and $\mathbf{w}_{3 \mathrm{e}}$, respectively. Thus, equation (48) consists of $p_{T}=n_{1}+2 n_{2}+2 p_{3}+n_{4}$ simultaneous equations, which are solved to give the unknown modal amplitudes and displacements.

\section{EXPERIMENT}

The theoretical model is validated here by comparing predictions against experimental measurements. The experimental methodology is the same as that described by Kirby et al. [1] and so only a brief overview is given here. The steel pipe to be analysed is a 3 inch Schedule 40 pipe, with $a_{1}=39 \mathrm{~mm}$ and $b_{1}=44.65 \mathrm{~mm}$. The pipe has an overall length of $6 \mathrm{~m}$ and an axisymmetric defect is machined into the pipe at a distance of $0.8 \mathrm{~m}$ from the far end of the pipe. Experiments 
are performed both with and without a coating on the pipe, with a coating of length $L_{c}=0.995 \mathrm{~m}$ added upstream of the defect and a length of $0.8 \mathrm{~m}$ covering the downstream pipe section, see Fig. 3. The pipe is excited by a commercial Teletest device [7] and for longitudinal modes the device is designed to excite the $\mathrm{L}(0,2)$ mode only, with the energy imparted into the $\mathrm{L}(0,1)$ mode minimised as far as possible. The $\mathrm{L}(0,2)$ mode is excited by 16 transducers spaced equally around the pipe circumference, with the transducers located $5.1 \mathrm{~m}$ from the defect (in region 1). An incident pulse consisting of 10 cycles of a Hann modulated tone burst signal is used, with a centre excitation frequency that may be varied from $20 \mathrm{kHz}$ to $120 \mathrm{kHz}$ in $5 \mathrm{kHz}$ steps. For each frequency step, each incident and reflected pulse was collected 64 times and averaged using the second Teletest monitoring tool, which uses four equally spaced transducers. For the uncoated pipe these transducers were placed $1 \mathrm{~m}$ from the defect, for the coated pipe they were placed $1 \mathrm{~m}$ from the coating. In general, the experiments were conducted over a frequency range with relatively low levels of dispersion; however, in order to minimise the error from dispersive effects the reflection coefficient was calculated in the frequency domain using an FFT with a rectangular time window. The width of each time window was carefully chosen to be wider than the length of each pulse to ensure the full capture of the signal in the time domain when dispersive effects were present. A large number of repetitive tests were undertaken for the $\mathrm{L}(0,2)$ mode, each with a frequency bandwidth of $125 \mathrm{~Hz}$. This bandwidth corresponds to the FFT step used to transfer the time into the frequency domain. Intermediate data are then removed so that a frequency bandwidth of 1.25 $\mathrm{kHz}$ is presented in the figures that follow in order to provide clarity for the experimental data.

The coating material used here was bitumen with a thickness of $1.5 \mathrm{~mm}$. Following $[1,2]$ the velocity of the bulk shear wave within the (viscoelastic) coating is written as

$$
c_{\mathrm{c}}=\frac{1}{\left[\frac{1}{\tilde{c}_{\mathrm{c}}}+i \frac{\alpha_{c}}{\omega}\right]} .
$$


Similarly, for the bulk longitudinal wave

$$
a_{\mathrm{c}}=\frac{1}{\left[\frac{1}{\tilde{a}_{\mathrm{c}}}+i \frac{\alpha_{L}}{\omega}\right]} .
$$

Here, $\tilde{c}_{\mathrm{c}}$ and $\tilde{a}_{\mathrm{c}}$ are the phase velocities for the bulk shear and longitudinal wave within the coating, and $\alpha_{\mathrm{c}}$ and $\alpha_{\mathrm{L}}$ denote the attenuation of the shear and longitudinal waves (in Nepers $/ \mathrm{m}$ ), respectively. Values for $\tilde{c}_{\mathrm{c}}$ and $\tilde{\alpha}_{\mathrm{c}}=\alpha_{\mathrm{c}} / \omega$ were investigated by Kirby et al. [1] and it was shown that the attenuation of the $\mathrm{T}(0,1)$ mode was very sensitive to values chosen for $\tilde{\alpha}_{\mathrm{c}}$. Clearly, the shear properties of the viscoelastic material also influence the behaviour of the longitudinal modes, and so in the next section the shear properties reported in [1] will be reviewed.

\section{RESULTS AND DISCUSSION}

In the results that follow, predicted and measured values for the reflection coefficient in region 1 are compared to one another. The reflection coefficient $\Lambda$ for mode $n$ is defined as

$$
\Lambda^{n}=\frac{B_{1}^{n}}{A_{1}^{1}}
$$

where $A_{1}^{1}$ denotes excitation of the $\mathrm{L}(0,2)$ mode.

\subsection{Uncoated Pipe}

Predictions for an uncoated pipe are readily obtained by setting $\alpha=\beta=0$ in section 2. Mode matching predictions are first compared against experimental data, which is carried out for a uniform defect with $a_{2}=41.85 \mathrm{~mm}$ (a $50 \%$ area reduction) and lengths of $L_{N}=15 \mathrm{~mm}$ and $L_{N}=$

$30 \mathrm{~mm}$; values of $c_{\mathrm{s}}=3260 \mathrm{~m} / \mathrm{s}, a_{\mathrm{s}}=5960 \mathrm{~m} / \mathrm{s}$ and $\rho=8030 \mathrm{~kg} / \mathrm{m}^{3}$ are used for the steel 
pipe [1]. In Fig. 4 mode matching predictions for $\Lambda^{0}$ and $\Lambda^{1}$ are compared against experimental measurements for $L_{N}=15 \mathrm{~mm}$, and here the mode matching model required 10 quadratic finite elements to achieve convergence to two decimal places at an upper frequency limit of $150 \mathrm{kHz}$. Note that the number of elements required is slightly larger than that used for the torsional modes, this is because it is necessary to study higher order longitudinal modes rather than the fundamental torsional mode. The solution of two equations now increases the number of degrees of freedom for the problem to $n_{t}=128$ (with $n_{2}=26$ and $n_{3}=14$ ), although the size of this problem is still very small and so it is still possible to obtain very quick solutions for longitudinal modes.

It is evident in Fig. 4 that good agreement between prediction and experiment is observed for the $\mathrm{L}(0,2)$ mode across a relatively wide frequency range. It is also interesting to note that significant energy scatters from the incident $\mathrm{L}(0,2)$ mode into the reflected $\mathrm{L}(0,1)$ mode, although this behaviour is seen to be strongly frequency dependent. In Fig. 5 the refection coefficient is presented for $L_{N}=30 \mathrm{~mm}$ using the same mesh as in the previous example, and again one observes good agreement between prediction and experiment. Some minor discrepancies are observed here at higher frequencies, which may be the result of experimental error. The most likely cause is transient uncertainties in the sensitivity of the transducers used in the Teletest monitoring tool. Here, it is interesting to note the strong influence that notch length has on the reflection of the $\mathrm{L}(0,1)$ and $\mathrm{L}(0,2)$ modes; this behaviour is similar to that seen for $\mathrm{T}(0,1)$ modes $[1,26]$ and it is clear that one must carefully choose an appropriate frequency range in order to be sure strong reflections are captured from a particular defect. The comparison between prediction and experiment in Figs. 4 and 5 serves to validate the uncoated predictions presented here. One may, of course, further investigate model validity by undertaking a sound power balance for the predictions presented in Figs. 4 and 5, and here the error in the sound power is less than $0.001 \%$ over the entire frequency range. It is interesting to note that this is not as good as that seen for the 
torsional modes [1], and this difference is probably caused by the extra difficulty of solving two coupled equations for the longitudinal modes, whereas only a single equation is required for the torsional modes.

It is sensible further to compare predictions with data found in the literature. However, very few studies have appeared for uniform axisymmetric defects, especially in the form of reflection coefficients covering a wide frequency range, and so further validation of the longitudinal model is undertaken here only for an axisymmetric elliptical (non uniform) defect using the data reported by Zhou et al. [27]. This presents a rigorous test of the model, as well as allowing comparison over a wide frequency range; however, the study of an elliptical (non-uniform) defect also requires the use of the hybrid method discussed in section 2.3. Accordingly, in Fig. 6 the refection coefficients for the $\mathrm{L}(0,1)$ and $\mathrm{L}(0,2)$ modes are presented for the an elliptical defect of exactly the same dimensions as that studied by Zhou et al. [27]. The data in Fig. 6 were obtained using a value of $p_{T}=1926$ (with $n_{2}=26$ ) over the entire frequency range, apart from the frequencies between the two resonant peaks that lie at approximately $k a=2.8$ and $k a=3.0$, where a value of $p_{T}=$ 7302 was used with $n_{2}=50$. The value of $p_{T}=1926$ was optimised for the highest frequency in Fig. 6 and so will be computationally less efficient at lower frequencies, although this can easily be rectified by adapting the size of the mesh with frequency. It is clear that a comparison between Fig. 6 and the results in Fig. 9 of ref. [27] shows almost identical predictions over a very wide frequency range (the upper limit in Fig. 6 corresponds to a frequency of $900 \mathrm{kHz}$ ). The sharp peaks seen in the reflection coefficients occur close to the cut-on frequency of higher order longitudinal modes. The only region where comparison is not so good is between the two resonant peaks at $k a=2.8$ and $k a=3.0$. It was noticeable that this frequency range required a significantly larger number of elements than that of the surrounding region in order to obtain a converged solution for the reflection coefficient (to two decimal places) and it may be that in the paper by Zhou et al. [27] insufficient degrees of freedom were used and some of the very complex behaviour over this 
frequency range was not captured. Nevertheless, it is reasonable to conclude here that the generally good agreement between Fig. 6 and the data reported by Zhou et al. provides confidence in the validity of the current model. Moreover, for the wide frequency range presented in Fig. 6, the average error in the sound power was $0.033 \%$, with a maximum value of $0.192 \%$, which further illustrates the accuracy of the method. Of course, it is common in long range ultrasonic testing to use much lower frequencies and so the number of degrees of freedom required here to demonstrate agreement with the data published by Zhou et al. [27] may be significantly lowered once the upper frequency limit is reduced. Furthermore, in common with the torsional modes, one may locate planes $\mathrm{B}$ and $\mathrm{C}$ very close to the defect in order to minimise computational expenditure.

Elliptical defects are rather difficult to machine accurately and so to further validate the hybrid method against measurement a tapered defect of the type discussed in ref. [1] is studied here. This type of defect is also more straightforward to model and for an upper frequency limit of $150 \mathrm{kHz}$ it is possible to reduce the number of element required to 38 , with $p_{T}=338$ and $n_{2}=18$. Of course, one may reduce this figure even further if the upper frequency limit is reduced and so for modest frequency ranges and simple defect geometries the hybrid method is capable of delivering very fast solutions and does not require large amounts of computer memory. It is interesting also to note that for longitudinal modes the number of elements required is greater than for the fundamental torsional mode, which is to be expected, but this increase is likely to be modest for relatively simple defects that are limited in length. However, it is clear that if one attempts to discretise long lengths of pipe then the study of higher order longitudinal modes is likely to be more expensive when compared to a fundamental torsional mode.

In Fig. 7 the reflection coefficients for four defects are plotted: a uniform defect of length $L_{N}=$ $15 \mathrm{~mm}$, a tapered defect with a taper angle of $\gamma=30^{\circ}$ (see Fig. 2), and two "V" shaped defects one with $\gamma=30^{\circ}$ and one with $\gamma=11^{\circ}$. The "V" shaped defects are obtained by using a very 
small defect length of $L_{N}=0.01 \mathrm{~mm}$, and for each non uniform defect $p_{T}=402$ and $n_{2}=26$.

Fig. 7 illustrates the influence of the taper on the reflection coefficient of the defect. This behaviour is similar to that seen for torsional modes [1], in which a small uplift for reflection coefficient is observed at lower frequencies when $\gamma=30^{\circ}$. Again, the refection coefficient is generally seen to drop for the "V" shaped defect, although Fig. 7 indicates that behaviour depends on the taper angle, and at higher frequencies it is possible to see an increase in the reflection coefficient from the larger taper angle.

In Fig. 8 the predicted reflection coefficient of a tapered defect with length $L_{N}=15 \mathrm{~mm}$ and a taper angle of $\gamma=30^{\circ}$ is compared against measurement, and here generally good agreement between the two is observed. It is, however, noticeable that the predictions tend to overestimate the reflection coefficient, a behaviour that was also observed to a lesser extent for the uniform notch in Fig. 4. It is possible here that the (idealised) numerical model, which does not account for additional energy losses in the system, is overestimating the amount of energy returning from the defect.

\subsection{Coated pipe}

In Figs. 9(a) and 9(b), a comparison between prediction and experiment is shown for a pipe coated with bitumen, see section 3 for details on the coating. The pipe contains a uniform defect that has a depth of $50 \%$ of the overall steel wall thickness, and a length $L_{N}=15 \mathrm{~mm}$ in Fig. 9(a), and $L_{N}=$ $30 \mathrm{~mm}$ in Fig. 9(b). For a uniform defect, the mode matching method is used to generate predictions, with convergence to two decimal places achieved at $150 \mathrm{kHz}$, with $n_{t}=220, n_{1}=$ $34, n_{2}=50$ and $n_{3}=18$. To compare predictions with experiment it is necessary to identify values for the bulk acoustic properties of the coating. Predictions are generated here using data for the shear properties reported by Kirby et al. [1] when studying $\mathrm{T}(0,1)$, with $\tilde{\alpha}_{c}=3.9 \times$ $10^{-3} \mathrm{~s} / \mathrm{m}, \tilde{c}_{c}=750 \mathrm{~m} / \mathrm{s}$, and $\rho_{\mathrm{c}}=1200 \mathrm{~kg} / \mathrm{m}^{3}$. For both figures, the values for the longitudinal 
properties reported by Barshinger and Rose [2] are used, so that $\tilde{a}_{c}=1860 \mathrm{~m} / \mathrm{s}$, and $\tilde{\alpha}_{L}=$ $\alpha_{L} / \omega=0.023 \times 10^{-3} \mathrm{~s} / \mathrm{m}$. It is apparent in Figs. 9(a) and (b) that the shear properties identified by Kirby et al. [1], when combined with the longitudinal properties of Barshinger and Rose [2], give good agreement with measured data and the quality of this agreement is at least comparable to that seen for uncoated pipes. The data used here for the shear properties of bitumen were obtained by comparison between prediction and experiment for the torsional $\mathrm{T}(0,1)$ mode only. It is shown in Figs. 9(a) and (b) that this data is also capable of predicting the behaviour of the $L(0,2)$ mode without any further amendment. This is an important result, as it provides an independent validation of the values obtained by Kirby et al. [1], and demonstrates that those values obtained deliver a good representation of the true acoustic performance of the material. Furthermore, Figs. 9(a) and 9(b) clearly demonstrate that the behaviour of the longitudinal modes is dominated by the shear properties of the coating; this has also been observed for flat plates, see for example Siminoetti [8]. Therefore, the results presented here show that $\tilde{\alpha}_{c}$ plays a very important role in both torsional and longitudinal elastic wave propagation, and it is vital that this quantity is accurately identified before attempting to model sound propagation in viscoelastic coatings. The dependence of the $\mathrm{L}(0,2)$ modes on the shear properties of a viscoelastic material has ramifications for the trial and error procedure originally carried out for $\mathrm{T}(0,1)$ modes by Kirby et al. [1]. Those results presented here demonstrate that the trial and error procedure should also include the longitudinal modes. This is because it is possible to assign material values that provide good agreement between prediction and experiment for the $\mathrm{T}(0,1)$ mode, but do not necessarily provide good agreement for the $\mathrm{L}(0,2)$ mode. Thus, the trial and error methodology should encompass both the $\mathrm{T}(0,1)$ and $\mathrm{L}(0,2)$ modes and only when good agreement is found for both can one be confident that the appropriate properties have been assigned. Of course, the advantage of the method proposed here is that it measures the material properties using guided waves and this is likely to give a good estimation of the true performance of the material, at least over the typical frequency range used in LRUT. 
It is interesting also to review the sensitivity of the reflection coefficients to changes in the longitudinal properties of the material. Accordingly, in Fig. 10, the reflection coefficient for a uniform defect of length $L_{N}=15 \mathrm{~mm}$ is plotted for different values of the longitudinal parameters $\tilde{\alpha}_{L}$ and $\tilde{a}_{\mathrm{c}}$, whist fixing the shear parameters $\tilde{\alpha}_{\mathrm{c}}$ and $\tilde{c}_{\mathrm{c}}$. Here, those values for $\tilde{\alpha}_{L}$ and $\tilde{a}_{\mathrm{c}}$ seen previously $\left(\tilde{\alpha}_{L}=0.023 \times 10^{-3} \mathrm{~s} / \mathrm{m}\right.$ and $\left.\tilde{a}_{c}=1860 \mathrm{~m} / \mathrm{s}\right)$ are used as a base from which to compare combinations of $10 \tilde{\alpha}_{L}, \tilde{\alpha}_{L} / 10,2 \tilde{c}_{L}$ and $\tilde{c}_{L} / 2$. It is immediately obvious in Fig. 10 that the reflection coefficients for $\mathrm{L}(0,2)$ are relatively insensitive to the longitudinal bulk acoustic properties of the coating, even for relatively large variations in the longitudinal properties. This behaviour is in contrast to that seen for the shear properties. For example, in Fig. 11 the values of $\tilde{\alpha}_{\mathrm{c}}=0.24 \times 10^{-3}$ and $\tilde{c}_{\mathrm{c}}=750 \mathrm{~m} / \mathrm{s}$ are perturbed by $\pm 10 \%$, which represents a much smaller range than the exercise conducted in Fig. 10, and here it is obvious how a relatively small change in the estimated shear bulk acoustic properties can impact significantly on the predicted values for the longitudinal reflection coefficient. Thus, Figs. 10 and 11 illustrate the importance of accurately identifying the shear properties of a viscoelastic coating.

The methodology described above for identifying the bulk acoustic properties assumes that any reflections from plane A are negligible. This assumption can be investigated theoretically by setting all of the modal amplitudes in the mode matching model equal to zero, except for the incident and reflected modes in region 1, and the incident mode in region 2. In Fig. 12, the reflection coefficient of plane $\mathrm{A}$ is plotted against the non-dimensional variable $k_{\mathrm{p}} t_{\mathrm{p}}$ for the following values of coating thickness: $t_{\mathrm{c}} / t_{\mathrm{p}}=0.25,0.5$ and 1 . This data was calculated using $n_{t}=44$, with $n_{1}=18$ and $n_{2}=26$. In Fig. 12 it is clear that the effect of the junction between the coated and uncoated region is negligible over the frequency range of interest provided one moves away from the cut-on frequency of the $\mathrm{L}(0,2)$ mode. It is noticeable, moreover, that plane A imparts less of an effect on the propagation of longitudinal modes when compared to torsional 
modes [1], although both sets of reflections coefficients indicate that the influence of plane A may successfully be ignored.

Finally, the hybrid method for the coated pipe is reviewed, and here the reflection coefficient for a tapered defect with a taper angle of $\gamma=30^{\circ}$ is shown for $L_{N}=15 \mathrm{~mm}$ in Fig. 13(a), and for $L_{N}=$ $30 \mathrm{~mm}$ in Fig. 13(b). The data used for the coated pipe are those optimised for a uniform defect, so that for the shear properties $\tilde{\alpha}_{c}=3.9 \times 10^{-3} \mathrm{~s} / \mathrm{m}, \tilde{c}_{c}=750 \mathrm{~m} / \mathrm{s}$, and for the longitudinal properties $\tilde{\alpha}_{\mathrm{L}}=0.023 \times 10^{-3} \mathrm{~s} / \mathrm{m}$ and $\tilde{a}_{\mathrm{c}}=1860 \mathrm{~m} / \mathrm{s}$, with $\rho_{c}=1200 \mathrm{~kg} / \mathrm{m}^{3}$. The data in Figs 13(a) and (b) were generated using 60 elements, so that $p_{T}=546$, with $n_{1}=18$ and $n_{2}=26$. In these figures generally good agreement between prediction and experiment is observed, although the reflection coefficient is over predicted for the $15 \mathrm{~mm}$ defect. These results do, however, give similar levels of agreement to that seen for uniform defects and so it is concluded here that the hybrid model is working successfully for a coated pipe.

\section{CONCLUSIONS}

A numerical model is presented here that is suitable for capturing the scattering of longitudinal modes from an axisymmetric defect in a coated pipe. By studying an axisymmetric problem it is shown that the modal scattering may be simplified to the extent that the longitudinal modes may be separated from the torsional ones, and computationally efficient solutions are obtained for uniform and non-uniform defects. Predictions are compared with experimental measurements for uniform and non-uniform defects and here good agreement between the two is observed for both coated and uncoated pipes. Moreover, this level of agreement is similar to that observed in a companion paper, see Kirby et al. [1]. 
Kirby et al. [1] deduced shear properties for a bitumen sample using an iterative trial and error technique based on comparing prediction and experiment. When these values are used in the theoretical model for longitudinal modes presented here, good agreement between prediction and experiment is observed. Thus, this article presents an independent validation of the shear properties for bitumen reported by Kirby et al. [1], and the good agreement observed here provides confidence in those values reported, at least up to a frequency of about $140 \mathrm{kHz}$. Furthermore, it is shown that the shear properties play a dominant role in the propagation of longitudinal modes and this means that the accurate identification of the bulk longitudinal properties is not so important and here the longitudinal data for bitumen reported by Barshinger and Rose [2] proved to be adequate. Accordingly, it may be concluded that it is very important to accurately identify the shear properties of a viscoelastic material before attempting to model guided wave propagation in coated pipes. However, it is demonstrated that a trial and error methodology for obtaining the shear properties of a viscoelastic material must include a comparison between prediction and experiment for the $\mathrm{L}(0,2)$ mode, as well as the $\mathrm{T}(0,1)$ mode. Only when good agreement is found for both modes can one conclude that the appropriate shear properties have been identified.

The advantage of the method presented here is that the shear properties may be obtained from a simple set of experiments using commercial hardware [7]. Moreover, the measurements are performed with guided waves on a coated pipe over the frequency range typically used in LRUT, which avoids the need for large bulk samples of the material to be available. It may, therefore, be possible to use this method in the field, maybe by using reflections from axisymmetric pipe discontinuities such as welds in order to infer the coating properties. Here, a weld may be modelled using the hybrid method, although the success of this method will depend on a priori knowledge of the weld geometry; the weld will also need to be sufficiently close to the measurement location so that the signal is of sufficient strength and quality to be able to implement the method described here. Furthermore, this assumes that one may characterise the coating material using constant 
values for the viscoelastic properties; this assumption is embedded in the use of a modal solution for regions in which the geometry and material properties are assumed to be uniform. In field based applications it is likely that the coatings will degrade over time and the viscoelastic properties will no longer be uniform. Accordingly, it remains to be seen if one can successfully use this methodology for characterising viscoelastic materials using field based measurements.

The results presented here demonstrate a methodology for obtaining the shear properties of a viscoelastic coating by comparing theoretical predictions to measurements obtained under laboratory conditions. The main aim of this work is to validate values for the shear properties of a viscoelastic material in the frequency range typically used in LRUT, and to use these values in the future development of theoretical models suitable for investigating improvements in experimental methodologies when capturing the scattering from non axisymmetric defects in coated pipes. 


\section{REFERENCES}

1. R. Kirby, Z. Zlatev, P. Mudge, On the scattering of torsional elastic waves from axisymmetric defects in coated pipes, Journal of Sound and Vibration 331 (2012) 3989-4004.

2. J.N. Barshinger, J.L.Rose, Guided wave propagation in an elastic hollow cylinder coated with a viscoelastic material, IEEE Transactions on Ultrasonics, Ferroelectrics, and Frequency Control 51 (2004) 1547-1556.

3. F. Simonetti, P. Cawley, Ultrasonic interferometry for the measurement of shear velocity and attenuation in viscoelastic solids, Journal of the Acoustical Society of America 115 (2004) $157-164$.

4. J. Ma, F. Simonetti, M.J.S. Lowe, Scattering of the fundamental torsional mode by a axisymmetric layer in a pipe, Journal of the Acoustical Society of America 120 (2006) 18711880.

5. M. Castaings, C. Bacon, Finite element modeling of torsional wave modes along pipes with absorbing materials, Journal of the Acoustical Society of America 119 (2006) 3741-3751.

6. D.N. Alleyne, M.J.S. Lowe, P. Cawley, The reflection of guided waves from circumferential notches in pipes, Journal of Applied Mechanics 65 (1998) 635-641.

7. <http://www.plantintegrity.com/teletest/mini-tools.jsp>

8. F. Simonetti, Sound propagation in lossless waveguides coated with attenuative materials, $\mathrm{PhD}$ Thesis, Imperial College, London (2003).

9. A. Marzani, E. Viola, I. Bartoli, F. Lanza di Scalea, P. Rizzo, A semi-analytical finite element formulation for modeling stress wave propagation in axisymmetric damped waveguides, Journal of Sound and Vibration 318 (2008) 488-505.

10. J. Hua, J.L. Rose, Guided wave inspection penetration power in viscoelastic coated pipes, Insight 52 (2010) 195-200. 
11. I. Bartoli, A. Marzani, F. Lanza di Scalea, E. Viola, Modeling wave propagation in damped waveguides of arbitrary cross-section, Journal of Sound and Vibration 295 (2006) 685-707.

12. R. Kirby, Transmission loss predictions for dissipative silencers of arbitrary cross section in the presence of mean flow, Journal of the Acoustical Society of America 114 (2003) 200-209.

13. T. Hayashi, K. Kawashima, Z. Sun, J.L. Rose, Analysis of flexural mode focusing by a semianalytical finite element method, Journal of the Acoustical Society of America 113 (2003) 1241-1248.

14. T. Hayashi, W-J. Song, J.L. Rose, Guided wave dispersion curves for a bar with an arbitrary cross-section, a rod and rail example, Ultrasonics 41 (2003) 175-183.

15. M. Castaings, M. Lowe, Finite element model for waves guided along solid systems of arbitrary section coupled to infinite solid media, Journal of the Acoustical Society of America 123 (2008) 696-708.

16. H.E. Engan, Torsional wave scattering from a diameter step in a rod, Journal of the Acoustical Society of America 104 (1998) 2015-2024.

17. J.J. Ditri, J.L. Rose, Excitation of guided elastic wave modes in hollow cylinders by applied surface tractions, Journal of Applied Physics 72 (1992) 2589-2597.

18. T. Vogt, M. Lowe, P. Cawley, The scattering of guided waves in partly embedded cylindrical structures, Journal of the Acoustical Society of America 113 (2003) 1258-1272.

19. A. Demma, P. Cawley, M. Lowe, Scattering of the fundamental shear horizontal mode from steps and notches in plates, Journal of the Acoustical Society of America 113 (2003) 18801891.

20. R. Kirby, Modeling sound propagation in acoustic waveguides using a hybrid numerical method, Journal of the Acoustical Society of America 124 (2008) 1930-1940.

21. M.V. Predoi, M. Castaings, L. Moreau, Influence of material viscoelasticity on the scattering of guided waves by defects, Journal of the Acoustical Society of America 124 (2008) 28832894. 
22. F. Benmeddour, F. Treyssède, L. Laguerre, Numerical modeling of guided wave interaction with non-axisymmetric cracks in elastic cylinders, International Journal of Solids and Structures, 48 (2011) 764-774.

23. J.B. Lawrie, An infinite, elastic, cylindrical shell with a finite number of ring constraints, Journal of Sound and Vibration 130 (1989) 189-206.

24. R.D. Gregory, A note on bi-orthogonality relations for elastic cylinders of general cross section, Journal of Elasticity 13 (1983) 351-355.

25. R. Kirby, A comparison between analytic and numerical methods for modelling automotive dissipative silencers with mean flow, Journal of Sound and Vibration 325 (2009) 565-582.

26. R. Carandente, J. Ma, P. Cawley, The scattering of the fundamental torsional mode from axisymmetric defects with varying depth profile in pipes, Journal of the Acoustical Society of America 127 (2010) 3440-3448.

27. W.J. Zhou, M.N. Ichchou, J.M. Mencik, Analysis of wave propagation in cylindrical pipes with local inhomogeneities, Journal of Sound and Vibration 319 (2009) 335-354. 


\section{APPENDIX 1}

Matrices for eigenvalue analysis in section 2.1.

$$
\begin{gathered}
\mathbf{K}_{0}^{\mathrm{p}}=\int_{a_{1}}^{b_{1}} \frac{\partial \mathbf{N}^{\mathrm{T}}}{\partial r} \frac{\partial \mathbf{N}}{\partial r} r d r+\left[\frac{2 c_{\mathrm{p}}^{2}}{a_{\mathrm{p}}^{2}}-1\right]\left(\left.\frac{1}{a_{1}}\right|_{r=a_{1}}-\left.\frac{1}{b_{1}}\right|_{r=b_{1}}\right) \\
\mathbf{K}_{0}^{\mathrm{c}}=\int_{b_{1}}^{b_{2}} \frac{\partial \mathbf{N}^{\mathrm{T}}}{\partial r} \frac{\partial \mathbf{N}}{\partial r} r d r+\left[\frac{2 c_{\mathrm{c}}^{2}}{a_{\mathrm{c}}^{2}}-1\right]\left(\left.\frac{1}{b_{1}}\right|_{r=b_{1}}-\left.\frac{1}{b_{2}}\right|_{r=b_{2}}\right) \\
\mathbf{K}_{1}^{\mathrm{p}}=\int_{a_{1}}^{b_{1}} \mathbf{N}^{\mathrm{T}} \frac{\partial \mathbf{N}}{\partial r} r d r, \quad \mathbf{K}_{1}^{\mathrm{c}}=\int_{b_{1}}^{b_{2}} \mathbf{N}^{\mathrm{T}} \frac{\partial \mathbf{N}}{\partial r} r d r \\
\mathbf{K}_{2}^{\mathrm{p}}=\int_{a_{1}}^{b_{1}} \frac{\mathbf{N}^{\mathrm{T}}}{r} \frac{\partial \mathbf{N}}{\partial r} r d r, \quad \mathbf{K}_{2}^{\mathrm{c}}=\int_{b_{1}}^{b_{2}} \frac{\mathbf{N}^{\mathrm{T}}}{r} \frac{\partial \mathbf{N}}{\partial r} r d r \\
\mathbf{K}_{3}^{\mathrm{p}}=\int_{a_{1}}^{b_{1}} \frac{\partial \mathbf{N}^{\mathrm{T}}}{\partial r} \frac{\partial \mathbf{N}}{\partial r} r d r, \quad \mathbf{K}_{3}^{\mathrm{c}}=\int_{b_{1}}^{b_{2}} \frac{\partial \mathbf{N}^{\mathrm{T}}}{\partial r} \frac{\partial \mathbf{N}}{\partial r} r d r \\
\mathbf{S}_{1}^{\mathrm{p}}=\int_{a_{1}}^{b_{1}} \frac{\mathbf{N}^{\mathrm{T}} \mathbf{N}}{r^{2}} r d r, \quad \mathbf{S}_{2}^{\mathrm{c}}=\int_{a_{1}}^{b_{1}} \frac{\mathbf{N}^{\mathrm{T}} \mathbf{N}}{r} r d r, \quad \mathbf{S}_{1}^{\mathrm{c}}=\int_{b_{1}}^{b_{2}} \frac{\mathbf{N}^{\mathrm{T}} \mathbf{N}}{r} r d r \\
\mathbf{S}_{0}^{\mathrm{p}}=\int_{a_{1}}^{b_{1}} \mathbf{\mathbf { N } ^ { \mathrm { T } } \mathbf { N }} r d r, \quad \mathbf{S}_{0}^{\mathrm{c}}=\int_{b_{1}}^{b_{2}} \mathbf{N}^{\mathrm{T}} \mathbf{N} r d r \\
\\
\end{gathered}
$$


Integrals for mode matching in section 2.2, and also for hybrid method in section 2.3.

$$
\begin{aligned}
& \mathrm{I}_{11 \pm}^{m n}=\int_{a_{1}}^{b_{1}}\left[\mp \tilde{T}_{r z 1}^{m}(r) u_{1}^{n}(r)-\tilde{T}_{z z 1}^{m}(r) w_{1}^{n}(r)\right] r d r \\
& \mathrm{I}_{12 \pm}^{m n}=\int_{a_{1}}^{b_{1}}\left[\mp \tilde{T}_{r z 1}^{m}(r) u_{2}^{n}(r)-\tilde{T}_{z z 1}^{m}(r) w_{2}^{n}(r)\right] r d r \\
& \mathrm{I}_{21 \pm}^{m n}=\int_{a_{1}}^{b_{1}}\left[u_{2}^{m}(r) \tilde{T}_{r z 1}^{n}(r) \mp w_{2}^{m}(r) \tilde{T}_{z z 1}^{n}(r)\right] r d r \\
& \mathrm{I}_{22 \pm}^{m n}=\int_{a_{1}}^{b_{1}}\left[u_{2}^{m}(r) \tilde{T}_{r z 2}^{n}(r) \mp w_{2}^{m}(r) \tilde{T}_{z z 2}^{n}(r)\right] r d r \\
& +\sigma \int_{b_{1}}^{b_{2}}\left[u_{2}^{m}(r) \tilde{T}_{r z 2}^{n}(r) \mp w_{2}^{m}(r) \tilde{T}_{z z 2}^{n}(r)\right] r d r \\
& \mathrm{I}_{32 \pm}^{m n}=\int_{a_{1}}^{a_{2}}\left[\mp \tilde{T}_{r z 3}^{m}(r) u_{2}^{n}(r)+\tilde{T}_{z z 3}^{m}(r) w_{2}^{n}(r)\right] r d r \\
& \mathrm{I}_{33 \pm}^{m n}=\int_{a_{1}}^{a_{2}}\left[\mp \tilde{T}_{r z 3}^{m}(r) u_{3}^{n}(r)+\tilde{T}_{z z 3}^{m}(r) w_{3}^{n}(r)\right] r d r \\
& \mathrm{H}_{22 \pm}^{m n}=\int_{a_{1}}^{b_{1}}\left[-u_{2}^{m}(r) \tilde{T}_{r z 2}^{n}(r) \mp w_{2}^{m}(r) \tilde{T}_{z z 2}^{n}(r)\right] r d r \\
& +\sigma \int_{b_{1}}^{b_{2}}\left[-u_{2}^{m}(r) \tilde{T}_{r z 2}^{n}(r) \mp w_{2}^{m}(r) \tilde{T}_{z z 2}^{n}(r)\right] r d r \\
& \mathrm{H}_{23 \pm}^{m n}=\int_{a_{1}}^{a_{2}}\left[-u_{2}^{m}(r) \tilde{T}_{r z 3}^{n}(r) \mp w_{2}^{m}(r) \tilde{T}_{z z 3}^{n}(r)\right] r d r \\
& \mathrm{H}_{32+}^{m n}=\int_{a_{1}}^{a_{2}}\left[-\tilde{T}_{r z 3}^{m}(r) u_{2}^{n}(r)-\tilde{T}_{z z 3}^{m}(r) w_{2}^{n}(r)\right] r d r \\
& \mathrm{H}_{33 \pm}^{m n}=\int_{a_{1}}^{a_{2}}\left[\mp \tilde{T}_{r z 3}^{m}(r) u_{3}^{n}(r)-\tilde{T}_{z z 3}^{m}(r) w_{3}^{n}(r)\right] r d r
\end{aligned}
$$




$$
\begin{gathered}
\mathrm{J}_{22 \pm}^{m n}=\int_{a_{1}}^{b_{1}}\left[\tilde{T}_{z z 2}^{n}(r) w_{2}^{m}(r)(r) \mp \tilde{T}_{r z 2}^{n} u_{2}^{m}(r)\right] r d r \\
\quad+\sigma \int_{b_{1}}^{b_{2}}\left[\tilde{T}_{z z 2}^{n}(r) w_{2}^{m}(r)(r) \mp \tilde{T}_{r z 2}^{n} u_{2}^{m}(r)\right] r d r \\
\mathrm{~J}_{23 \pm}^{m n}=\int_{a_{1}}^{a_{2}}\left[u_{2}^{m}(r) \tilde{T}_{r z 3}^{n}(r) \mp w_{2}^{m}(r) \tilde{T}_{z z 3}^{n}(r)\right] r d r
\end{gathered}
$$

The integrals in Eqs. (A9) - (A20) are carried out numerically for $m=0,1, \ldots . n_{q}$ and $n=$ $0,1, \ldots . n_{q}$.

\section{APPENDIX 3}

Matrices for hybrid method in section 2.3.

$$
\begin{aligned}
& \mathbf{K}_{1 r}=\int_{\Omega_{3 \mathrm{p}}}\left[\frac{\partial \mathbf{R}^{\mathrm{T}}}{\partial r} \frac{\partial \mathbf{R}}{\partial r}+\frac{c_{\mathrm{p}}^{2}}{a_{\mathrm{p}}^{2}} \frac{\partial \mathbf{R}^{\mathrm{T}}}{\partial z} \frac{\partial \mathbf{R}}{\partial z}-\frac{\mathbf{R}^{\mathrm{T}}}{r} \frac{\partial \mathbf{R}}{\partial z}+\frac{\mathbf{R}^{\mathrm{T}} \mathbf{R}}{r^{2}}-\gamma_{\mathrm{p}}^{2} \mathbf{R}^{\mathrm{T}} \mathbf{R}\right] d \Omega_{3 \mathrm{p}} \\
& +\sigma \int_{\Omega_{3 c}}\left[\frac{\partial \mathbf{R}^{\mathrm{T}}}{\partial r} \frac{\partial \mathbf{R}}{\partial r}+\frac{c_{\mathrm{c}}^{2}}{a_{\mathrm{c}}^{2}} \frac{\partial \mathbf{R}^{\mathrm{T}}}{\partial z} \frac{\partial \mathbf{R}}{\partial z}-\frac{\mathbf{R}^{\mathrm{T}}}{r} \frac{\partial \mathbf{R}}{\partial z}+\frac{\mathbf{R}^{\mathrm{T}} \mathbf{R}}{r^{2}}-\gamma_{\mathrm{c}}^{2} \mathbf{R}^{\mathrm{T}} \mathbf{R}\right] d \Omega_{3 \mathrm{c}} \\
& +\left[2 \frac{c_{\mathrm{p}}^{2}}{a_{\mathrm{p}}^{2}}-1\right]\left\{\int_{\Gamma_{3 \mathrm{p}}} \frac{\mathbf{R}^{\mathrm{T}} \mathbf{R}}{r} \cdot \mathbf{n}_{r} d \Gamma_{3 \mathrm{p}}\right\}\left\{\mathbf{u}_{3 \mathrm{p}}\right\}+\sigma\left[2 \frac{c_{\mathrm{c}}^{2}}{a_{\mathrm{c}}^{2}}-1\right]\left\{\int_{\Gamma_{3 \mathrm{c}}} \frac{\mathbf{R}^{\mathrm{T}} \mathbf{R}}{r} \cdot \mathbf{n}_{r} d \Gamma_{3 \mathrm{c}}\right\}\left\{\mathbf{u}_{3 \mathrm{c}}\right\} \\
& \mathbf{K}_{1 z} \int_{\Omega_{3 \mathrm{p}}}\left[\frac{\partial \mathbf{Z}^{\mathrm{T}}}{\partial r} \frac{\partial \mathbf{Z}}{\partial z}-\frac{c_{\mathrm{p}}^{2}}{a_{\mathrm{p}}^{2}} \frac{\partial \mathbf{Z}^{\mathrm{T}}}{\partial z} \frac{\partial \mathbf{Z}}{\partial r}\right] d \Omega_{3 \mathrm{p}}+\sigma \int_{\Omega_{3 c}}\left[\frac{\partial \mathbf{Z}^{\mathrm{T}}}{\partial r} \frac{\partial \mathbf{Z}}{\partial z}-\frac{c_{\mathrm{c}}^{2}}{a_{\mathrm{c}}^{2}} \frac{\partial \mathbf{Z}^{\mathrm{T}}}{\partial z} \frac{\partial \mathbf{Z}}{\partial r}\right] d \Omega_{3 \mathrm{c}} \\
& -2 \frac{c_{\mathrm{p}}^{2}}{a_{\mathrm{p}}^{2}} \int_{\Gamma_{3 \mathrm{p}}}\left[\mathbf{R}^{\mathrm{T}} \frac{\partial \mathbf{Z}}{\partial r} \cdot \mathbf{n}_{z}-\mathbf{R}^{\mathrm{T}} \frac{\partial \mathbf{Z}}{\partial z} \cdot \mathbf{n}_{r}\right] d \Gamma_{3 \mathrm{p}}-2 \sigma \frac{c_{\mathrm{c}}^{2}}{a_{\mathrm{c}}^{2}} \int_{\Gamma_{3 \mathrm{c}}}\left[\mathbf{R}^{\mathrm{T}} \frac{\partial \mathbf{Z}}{\partial r} \cdot \mathbf{n}_{z}-\mathbf{R}^{\mathrm{T}} \frac{\partial \mathbf{Z}}{\partial z} \cdot \mathbf{n}_{r}\right] d \Gamma_{3 \mathrm{c}} \\
& \mathbf{K}_{2 z}=\int_{\Omega_{3 \mathrm{p}}}\left[\frac{c_{\mathrm{p}}^{2}}{a_{\mathrm{p}}^{2}} \frac{\partial \mathbf{Z}^{\mathrm{T}}}{\partial r} \frac{\partial \mathbf{Z}}{\partial r}+\frac{\partial \mathbf{Z}^{\mathrm{T}}}{\partial z} \frac{\partial \mathbf{Z}}{\partial z}-\frac{c_{\mathrm{p}}^{2}}{a_{\mathrm{p}}^{2}} \frac{\mathbf{Z}^{\mathrm{T}}}{r} \frac{\partial \mathbf{Z}}{\partial r}-\gamma_{\mathrm{p}}^{2} \mathbf{Z}^{\mathrm{T}} \mathbf{Z}\right] d \Omega_{3 \mathrm{p}} \\
& +\sigma \int_{\Omega_{3 c}}\left[\frac{c_{\mathrm{c}}^{2}}{a_{\mathrm{c}}^{2}} \frac{\partial \mathbf{Z}^{\mathrm{T}}}{\partial r} \frac{\partial \mathbf{Z}}{\partial r}+\frac{\partial \mathbf{Z}^{\mathrm{T}}}{\partial z} \frac{\partial \mathbf{Z}}{\partial z}-\frac{c_{\mathrm{c}}^{2}}{a_{\mathrm{c}}^{2}} \frac{\mathbf{Z}^{\mathrm{T}}}{r} \frac{\partial \mathbf{Z}}{\partial r}-\gamma_{\mathrm{c}}^{2} \mathbf{Z}^{\mathrm{T}} \mathbf{Z}\right] d \Omega_{3 \mathrm{c}}
\end{aligned}
$$




$$
\begin{gathered}
\mathbf{K}_{2 r}=\int_{\Omega_{3 \mathrm{p}}}\left[\frac{\partial \mathbf{R}^{\mathrm{T}}}{\partial z} \frac{\partial \mathbf{R}}{\partial r}-\frac{c_{\mathrm{p}}^{2}}{a_{\mathrm{p}}^{2}} \frac{\partial \mathbf{R}^{\mathrm{T}}}{\partial r} \frac{\partial \mathbf{R}}{\partial z}+\left(\frac{c_{\mathrm{p}}^{2}}{a_{\mathrm{p}}^{2}}-1\right) \frac{\mathbf{R}^{\mathrm{T}}}{r} \frac{\partial \mathbf{R}}{\partial z}\right] d \Omega_{3 \mathrm{p}} \\
\quad+\sigma \int_{\Omega_{3 c}}\left[\frac{\partial \mathbf{R}^{\mathrm{T}}}{\partial z} \frac{\partial \mathbf{R}}{\partial r}-\frac{c_{\mathrm{c}}^{2}}{a_{\mathrm{c}}^{2}} \frac{\partial \mathbf{R}^{\mathrm{T}}}{\partial r} \frac{\partial \mathbf{R}}{\partial z}+\left(\frac{c_{\mathrm{c}}^{2}}{a_{\mathrm{c}}^{2}}-1\right) \frac{\mathbf{R}^{\mathrm{T}}}{r} \frac{\partial \mathbf{R}}{\partial z}\right] d \Omega_{3 \mathrm{c}} \\
-2 \frac{c_{\mathrm{p}}^{2}}{a_{\mathrm{p}}^{2}} \int_{\Gamma_{3 \mathrm{p}}}\left[\mathbf{Z}^{\mathrm{T}} \frac{\partial \mathbf{R}}{\partial r} \cdot \mathbf{n}_{z}-\mathbf{R}^{\mathrm{T}} \frac{\partial \mathbf{R}}{\partial z} \cdot \mathbf{n}_{r}\right] d \Gamma_{3 \mathrm{p}}-2 \sigma \frac{c_{\mathrm{c}}^{2}}{a_{\mathrm{c}}^{2}} \int_{\Gamma_{3 \mathrm{c}}}\left[\mathbf{Z}^{\mathrm{T}} \frac{\partial \mathbf{R}}{\partial r} \cdot \mathbf{n}_{z}-\mathbf{R}^{\mathrm{T}} \frac{\partial \mathbf{R}}{\partial z} \cdot \mathbf{n}_{r}\right] d \Gamma_{3 \mathrm{c}} \\
-\left[2 \frac{c_{\mathrm{p}}^{2}}{a_{\mathrm{p}}^{2}}-1\right] \int_{\Gamma_{3 \mathrm{p}}} \frac{\mathbf{Z}^{\mathrm{T}} \mathbf{R}}{r} \cdot \mathbf{n}_{z} d \Gamma_{3 \mathrm{p}}-\sigma\left[2 \frac{c_{\mathrm{c}}^{2}}{a_{\mathrm{c}}^{2}}-1\right] \int_{\Gamma_{3 c}} \frac{\mathbf{Z}^{\mathrm{T}} \mathbf{R}}{r} \cdot \mathbf{n}_{z} d \Gamma_{3 \mathrm{c}} \\
\mathbf{Q}_{1}=\int_{\Gamma_{\mathrm{Xp}}} \tilde{T}_{r z_{\mathrm{p}}}^{n} \mathbf{R} d \Gamma_{\mathrm{Xp}}+\sigma \int_{\Gamma_{\mathrm{Xc}}} \tilde{T}_{r z_{\mathrm{c}}}^{n} \mathbf{R} d \Gamma_{\mathrm{Xc}}, \quad\left(n=0,1, \ldots . n_{2}\right) \\
\mathbf{R}_{2}=\int_{\Gamma_{\mathrm{Xp}}} \tilde{T}_{z z_{\mathrm{p}}}^{m} \mathbf{R} d \Gamma_{\mathrm{Xp}}+\sigma \int_{\Gamma_{\mathrm{Xc}}} \tilde{T}_{z z_{\mathrm{c}}}^{m} \mathbf{R} d \Gamma_{\mathrm{Xc}}, \quad\left(n=0,1, \ldots . n_{2}\right) \\
\int_{\Gamma_{\mathrm{Yp}}} \tilde{T}_{r z_{\mathrm{p}}}^{m} \mathbf{R} d \Gamma_{\mathrm{Yp}}+\sigma \int_{\Gamma_{\mathrm{Yc}}} \tilde{T}_{z z_{\mathrm{c}}}^{m} \mathbf{R} d \Gamma_{\mathrm{Yc}}, \quad\left(n=0,1, \ldots n_{2}\right) \\
\tilde{T}_{r \mathrm{Y}_{\mathrm{Y}}}^{m} \mathbf{R} d \Gamma_{\mathrm{Yc}}, \quad\left(n=0,1, \ldots . n_{2}\right)
\end{gathered}
$$




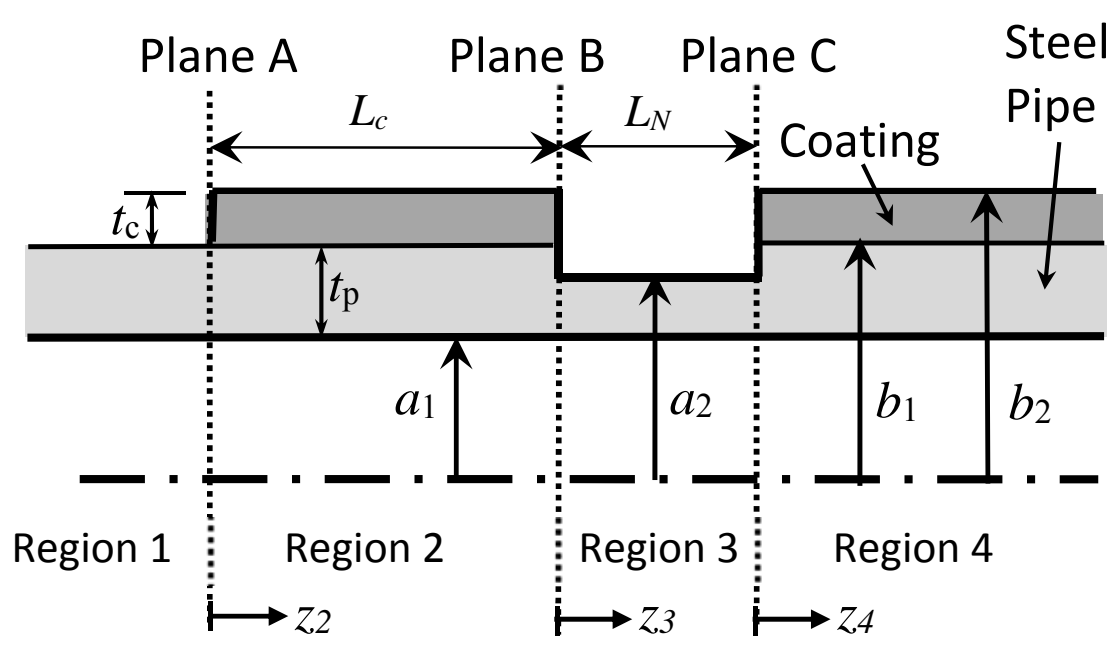

Figure 1. Geometry of uniform defect. 


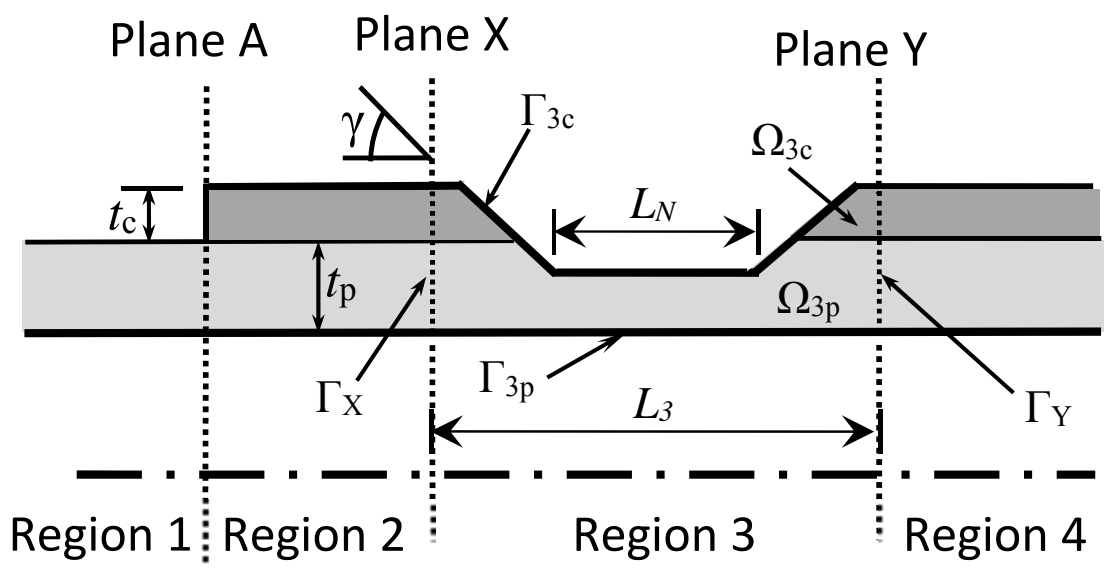

Figure 2. Geometry of non-uniform defect. 


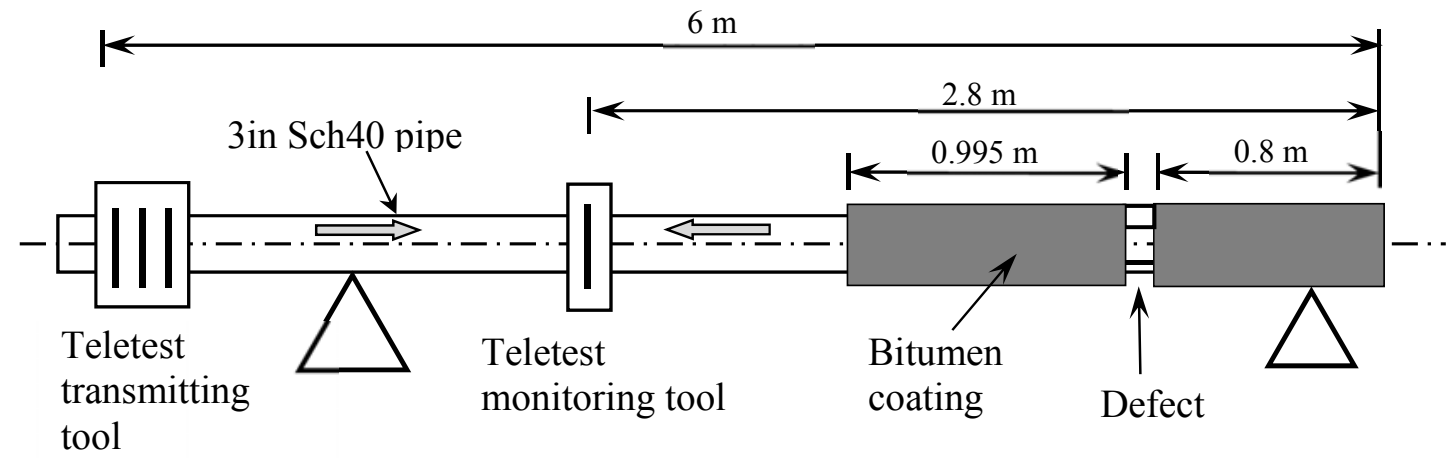

Figure 3. Experimental apparatus. 


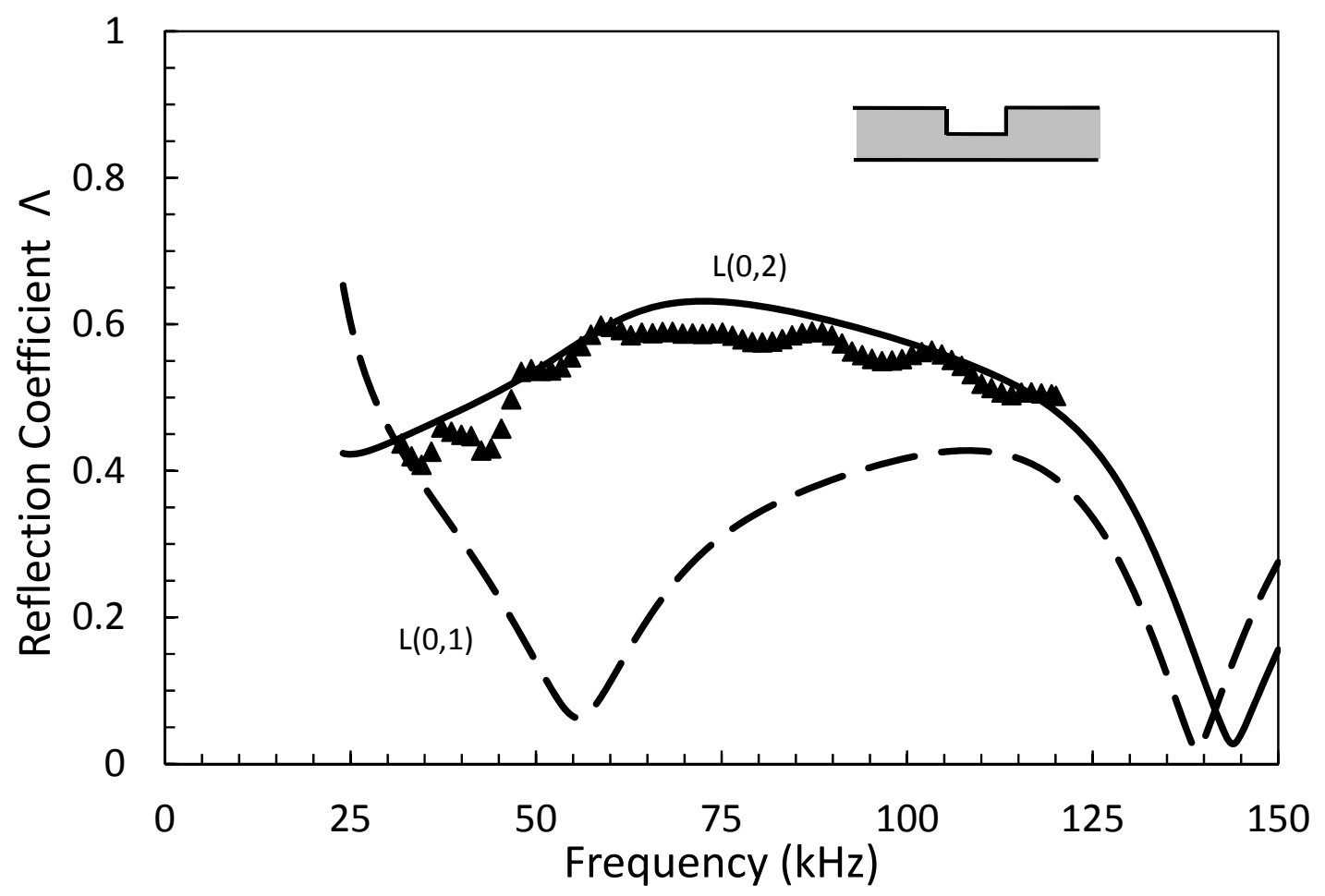

Figure 4. Mode matching predictions for uniform defect with $L_{N}=15 \mathrm{~mm}$.

$\longrightarrow, \mathrm{L}(0,2) ;----, \mathrm{L}(0,1) ; \boldsymbol{\Delta}$, experiment 


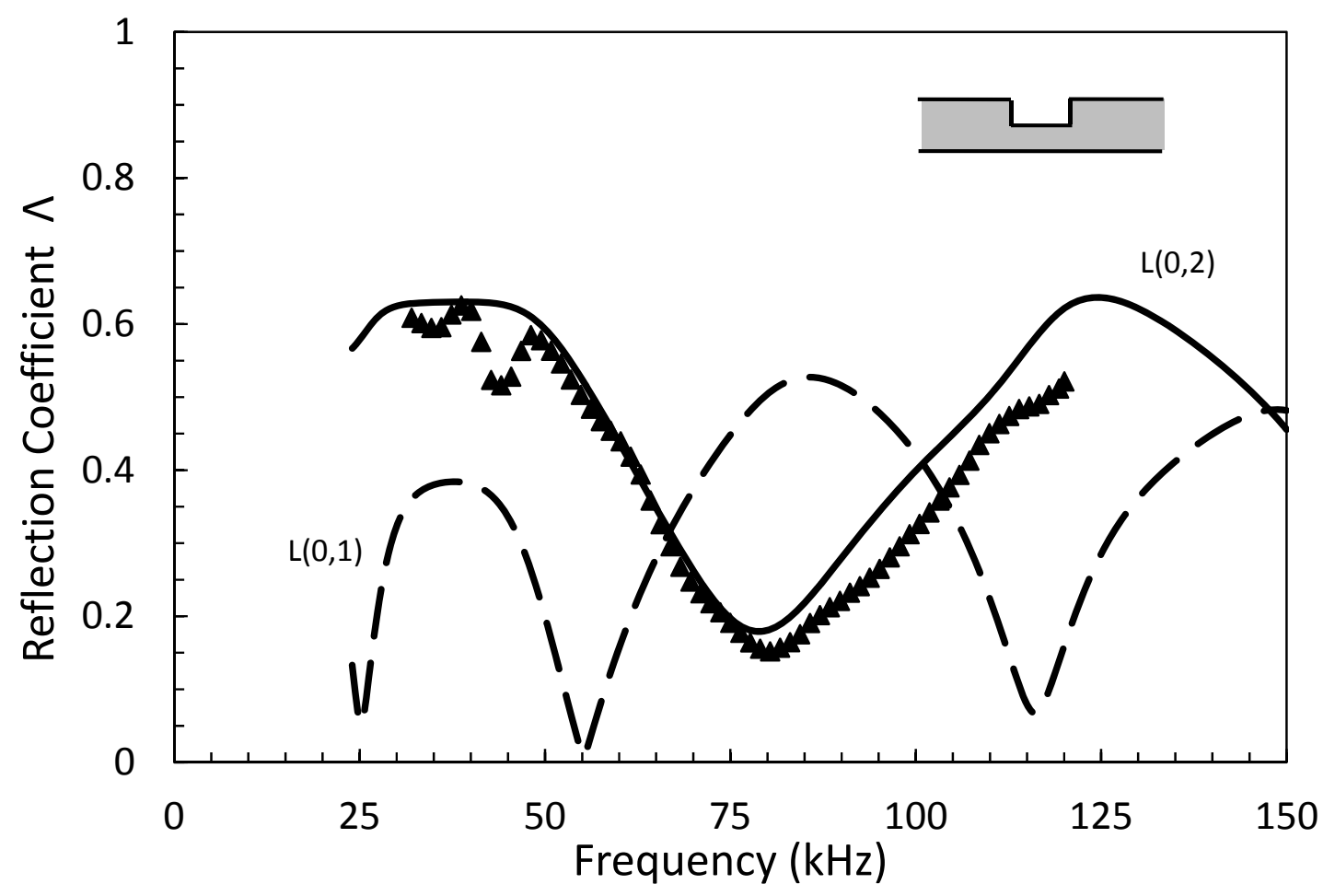

Figure 5. Mode matching predictions for uniform defect with $L_{N}=30 \mathrm{~mm}$.

$\longrightarrow, \mathrm{L}(0,2) ;----, \mathrm{L}(0,1) ; \boldsymbol{\Delta}$, experiment 


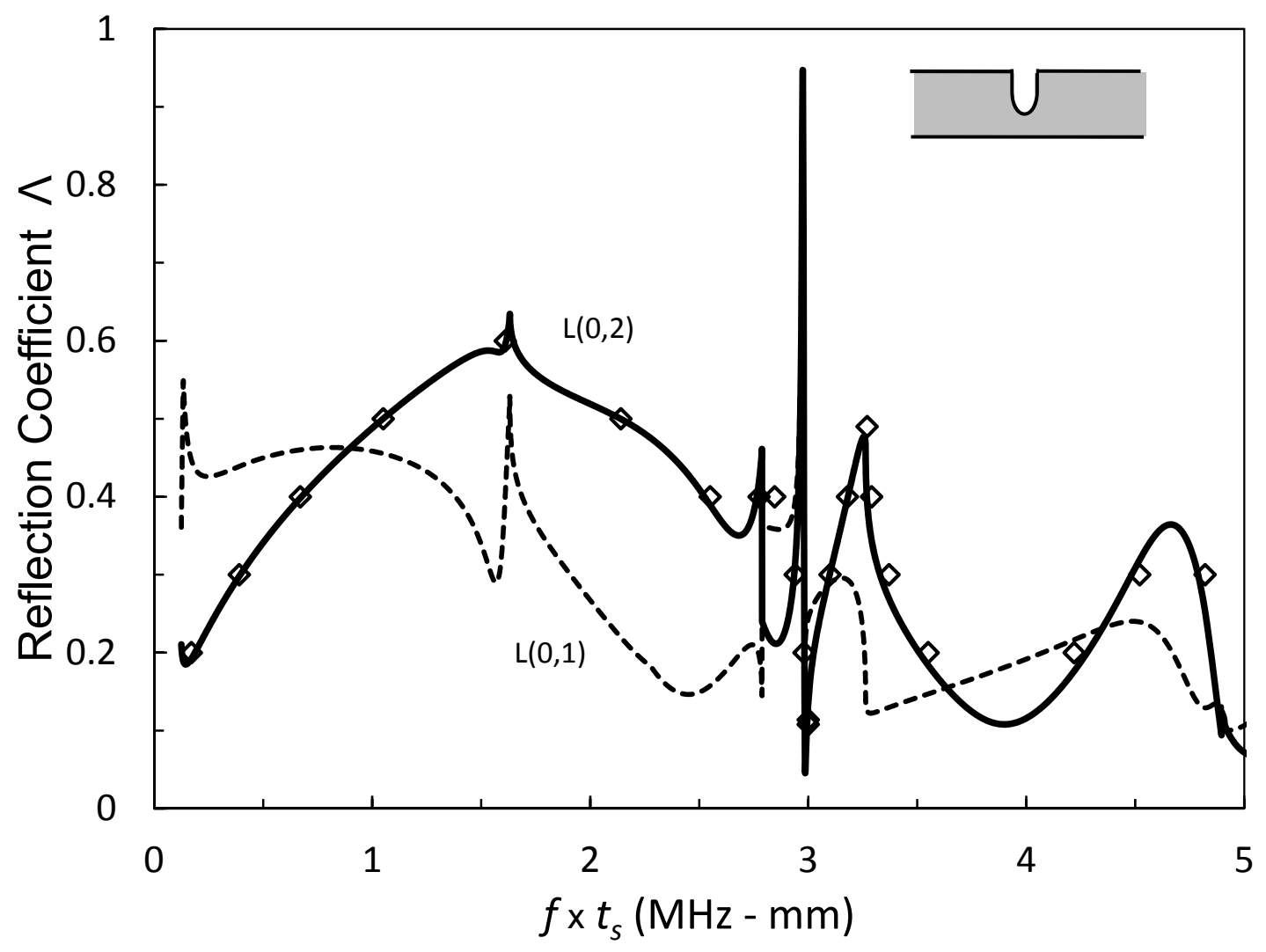

Figure 6. Hybrid method predictions for elliptical defect of Zhou et al. [25].

$\longrightarrow, \mathrm{L}(0,2) ;-\ldots-\_, \mathrm{L}(0,1) ; \diamond, \mathrm{L}(0,2)$ predictions by Zhou et al. [25]. 


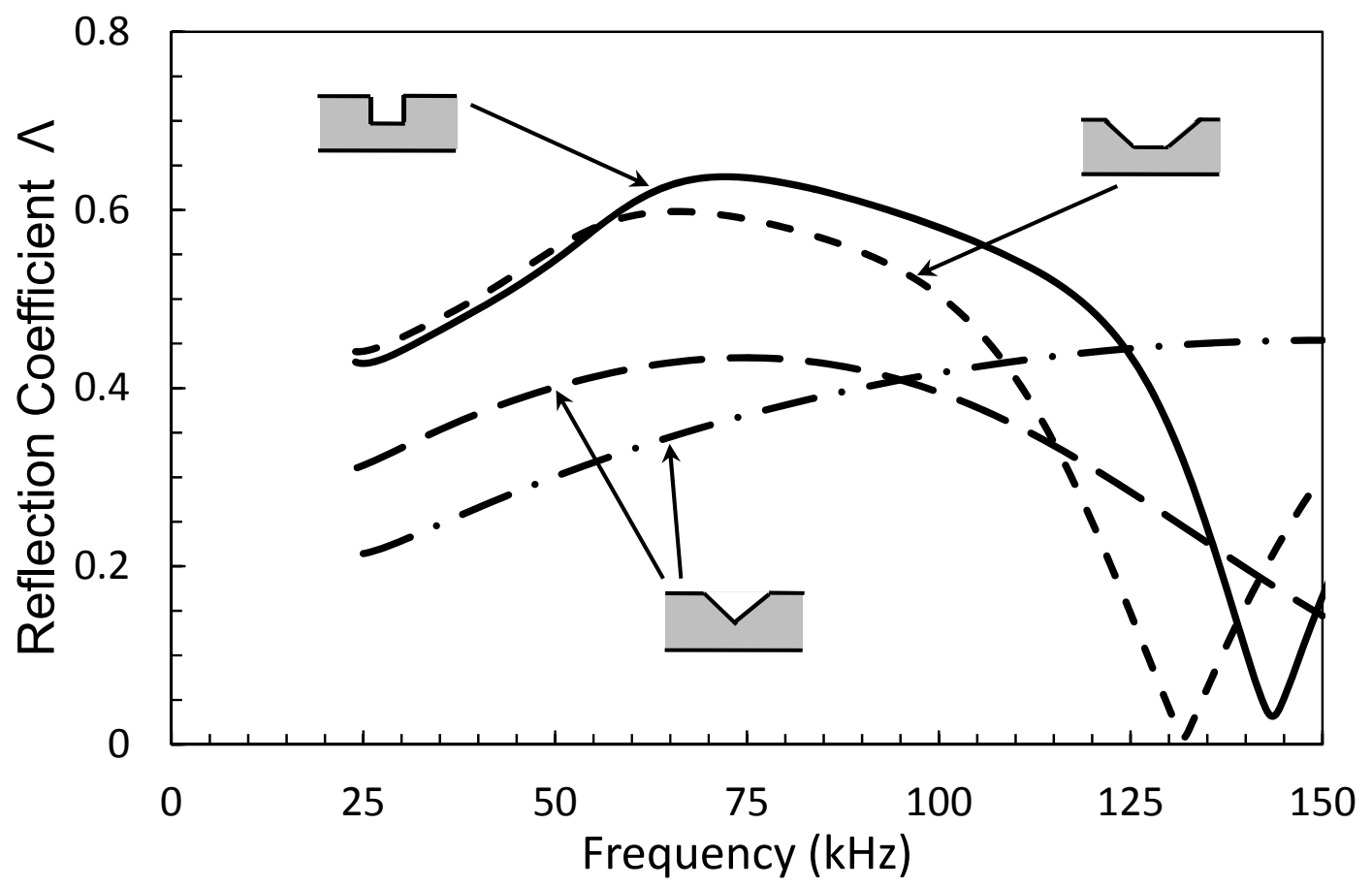

Figure 7. Hybrid method predictions for the reflection coefficient of $L(0,2)$ for uniform and nonuniform defects. - , uniform defect $L_{N}=15 \mathrm{~mm} ;----$, tapered defect, $\gamma=30^{\circ}$, $-\cdots \cdots$, "V" defect, $\gamma=30^{\circ},--\square$, ,V" defect, $\gamma=11^{\circ}$. 


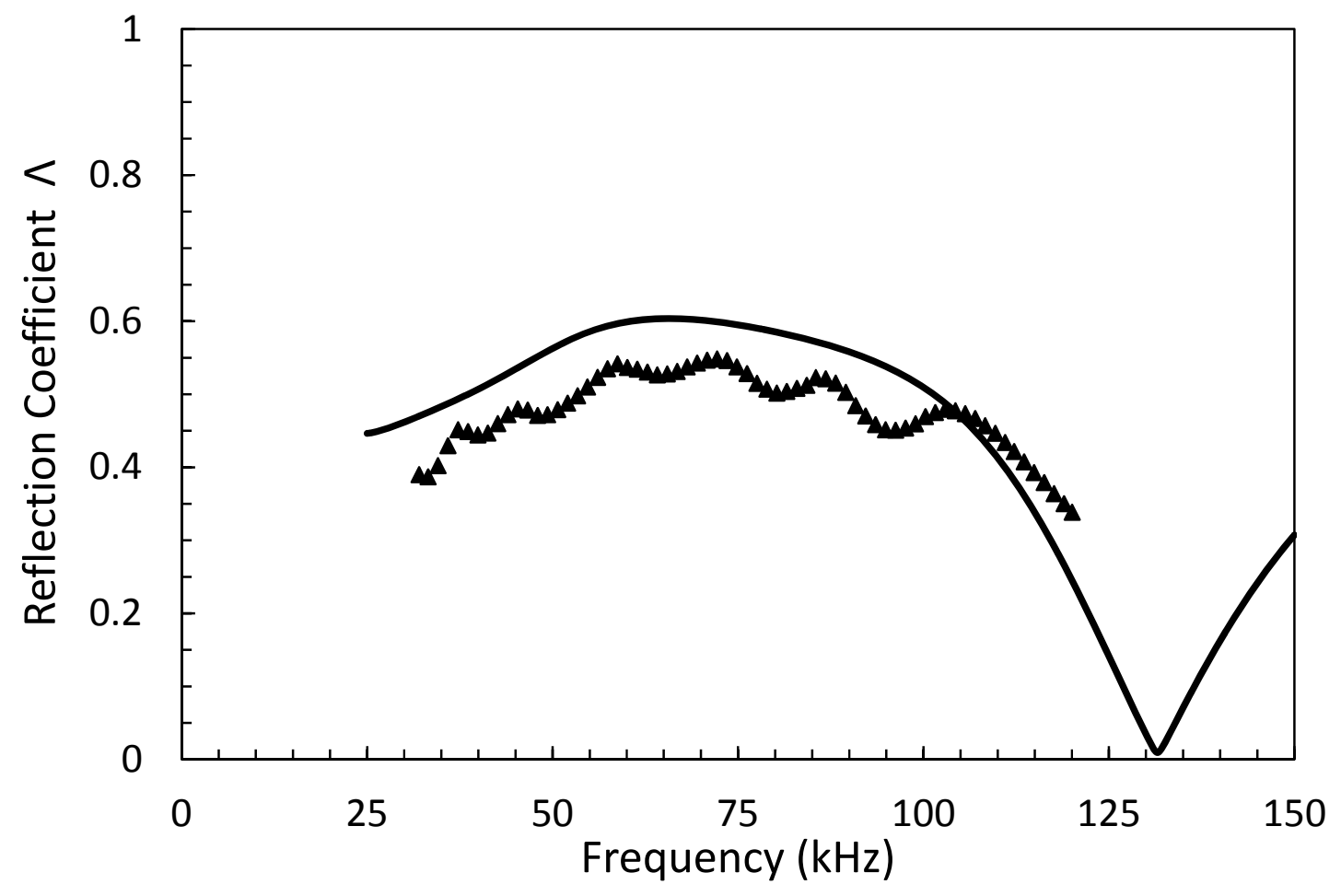

Figure 8. Hybrid method predictions for a tapered defect with $\gamma=30^{\circ}$ and $L_{N}=15 \mathrm{~mm}$ for $\mathrm{L}(0,2), . \quad-$, prediction; $\boldsymbol{\Lambda}$, experiment. 

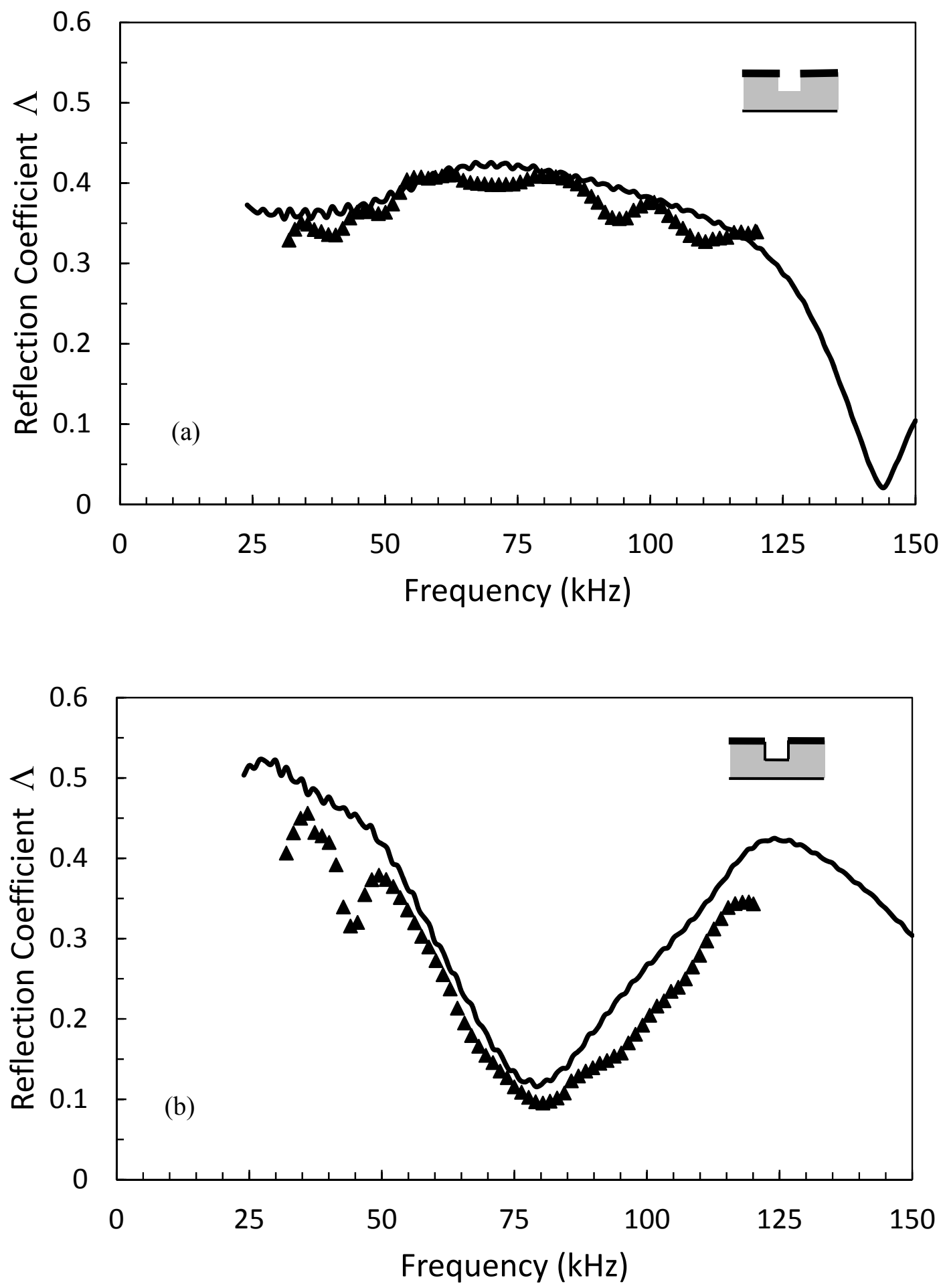

Figure 9. Mode matching predictions for $\mathrm{L}(0,2)$ for a uniform defect in a coated pipe with $\tilde{c}_{\mathrm{c}}=$ $750 \mathrm{~m} / \mathrm{s}, \tilde{a}_{\mathrm{c}}=1860 \mathrm{~m} / \mathrm{s}$ and $\tilde{\alpha}_{\mathrm{L}}=0.023 \times 10^{-3} \mathrm{~s} / \mathrm{m}$ : (a) $L_{N}=15 \mathrm{~mm}$, (b) $L_{N}=30 \mathrm{~mm}$. , $\tilde{\alpha}_{\mathrm{c}}=3.9 \times 10^{-3} \mathrm{~s} / \mathrm{m}, \rho_{\mathrm{c}}=1200 \mathrm{~kg} / \mathrm{m}^{3}[1] ; \boldsymbol{\Lambda}$, experiment. 


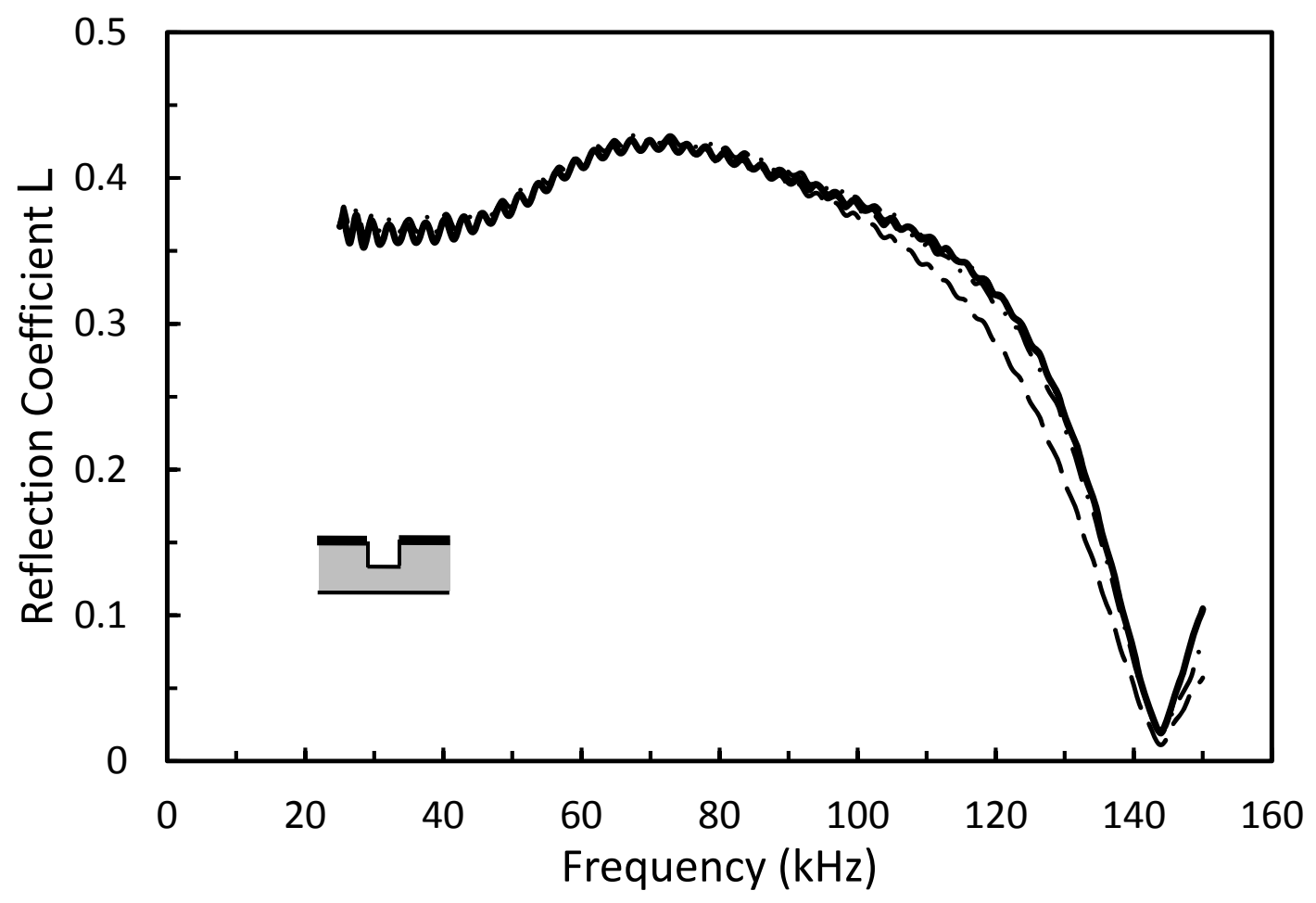

Figure 10. Mode matching predictions for $\mathrm{L}(0,2)$ for a uniform defect in a coated pipe, with

$$
\begin{gathered}
L_{N}=15 \mathrm{~mm}, \tilde{\alpha}_{L}^{\text {base }}=0.023 \times 10^{-3} \mathrm{~s} / \mathrm{m}, \tilde{a}_{\mathrm{c}}^{\text {base }}=1860 \mathrm{~m} / \mathrm{s} ; \text { and, } \tilde{\alpha}_{\mathrm{c}}=3.9 \times 10^{-3} \mathrm{~s} / \mathrm{m}, \tilde{c}_{\mathrm{c}}= \\
\quad 750 \frac{\mathrm{m}}{\mathrm{s}}, \rho_{\mathrm{c}}=1200 \mathrm{~kg} / \mathrm{m}^{3} .-\tilde{\alpha}_{L}=\tilde{\alpha}_{L}^{\text {base }}, \tilde{a}_{\mathrm{c}}=\tilde{a}_{\mathrm{c}}^{\text {base }} ; \\
---, \tilde{\alpha}_{L}=10 \tilde{\alpha}_{L}^{\text {base }}, \tilde{a}_{\mathrm{c}}=0.5 \tilde{a}_{\mathrm{c}}^{\text {base }} ;-\cdots-, \tilde{\alpha}_{L}=0.1 \tilde{\alpha}_{L}^{\text {base }}, \tilde{a}_{\mathrm{c}}=0.5 \tilde{a}_{\mathrm{c}}^{\text {base }} ; \\
---\tilde{\alpha}_{L}=0.1 \tilde{\alpha}_{L}^{\text {base }}, \tilde{a}_{\mathrm{c}}=2 \tilde{a}_{\mathrm{c}}^{\text {base }} ;-\cdots-, \tilde{\alpha}_{L}=10 \tilde{\alpha}_{L}^{\text {base }}, \tilde{a}_{\mathrm{c}}=2 \tilde{a}_{\mathrm{c}}^{\text {base }} .
\end{gathered}
$$




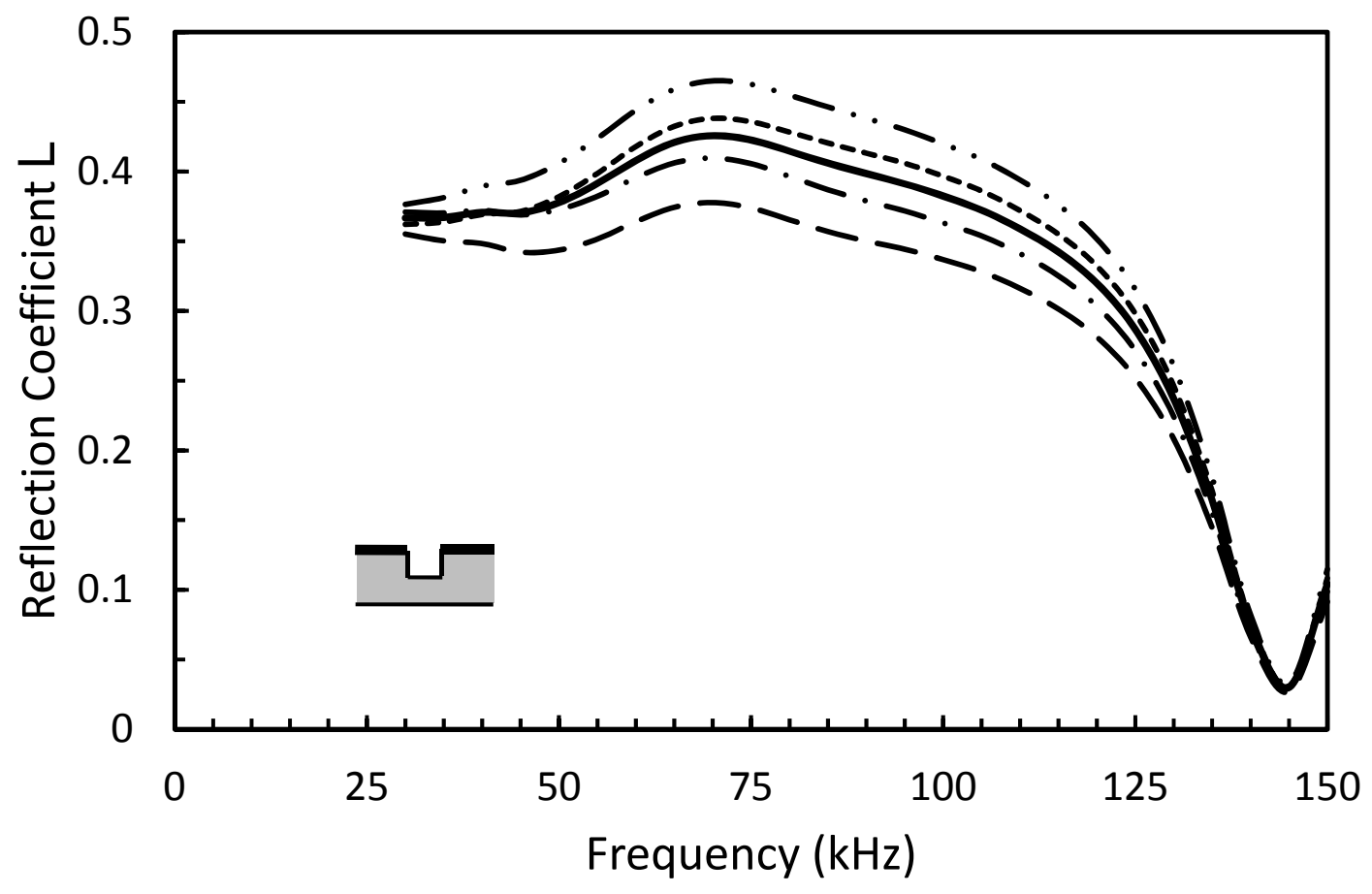

Figure 11. Mode matching predictions for $\mathrm{L}(0,2)$ for a uniform defect in a coated pipe, with

$$
\begin{gathered}
L_{N}=15 \mathrm{~mm}, \tilde{\alpha}_{\mathrm{c}}^{\text {base }}=3.9 \times 10^{-3} \mathrm{~s} / \mathrm{m}, \tilde{c}_{\mathrm{c}}^{\text {base }}=750 \mathrm{~m} / \mathrm{s}, \text { and } \tilde{\alpha}_{L}=0.24 \times 10^{-3} \mathrm{~s} / \mathrm{m}, \tilde{\alpha}_{c}= \\
1860 \mathrm{~m} / \mathrm{s} \text { and } \rho_{\mathrm{c}}=1200 \mathrm{~kg} / \mathrm{m}^{3} .-, \tilde{\alpha}_{\mathrm{c}}=\tilde{\alpha}_{\mathrm{c}}^{\text {base }}, \tilde{c}_{\mathrm{c}}=\tilde{c}_{\mathrm{c}}^{\text {base }} ; \\
--, \tilde{\alpha}_{\mathrm{c}}=0.9 \tilde{\alpha}_{\mathrm{c}}^{\mathrm{base}}, \tilde{c}_{\mathrm{c}}=0.9 \tilde{c}_{\mathrm{c}}^{\text {base }} ;-\cdot-\cdot \tilde{\alpha}_{\mathrm{c}}=0.9 \tilde{\alpha}_{\mathrm{c}}^{\text {base }}, \tilde{c}_{\mathrm{c}}=1.1 \tilde{c}_{\mathrm{c}}^{\text {base }} ; \\
---, \tilde{\alpha}_{\mathrm{c}}=1.1 \tilde{\alpha}_{\mathrm{c}}^{\text {base }}, \tilde{c}_{\mathrm{c}}=0.9 \tilde{c}_{\mathrm{c}}^{\text {base }} ;-\cdots-, \tilde{\alpha}_{\mathrm{c}}=1.1 \tilde{\alpha}_{\mathrm{c}}^{\text {base }}, \tilde{c}_{\mathrm{c}}=1.1 \tilde{c}_{\mathrm{c}}^{\text {base }} .
\end{gathered}
$$




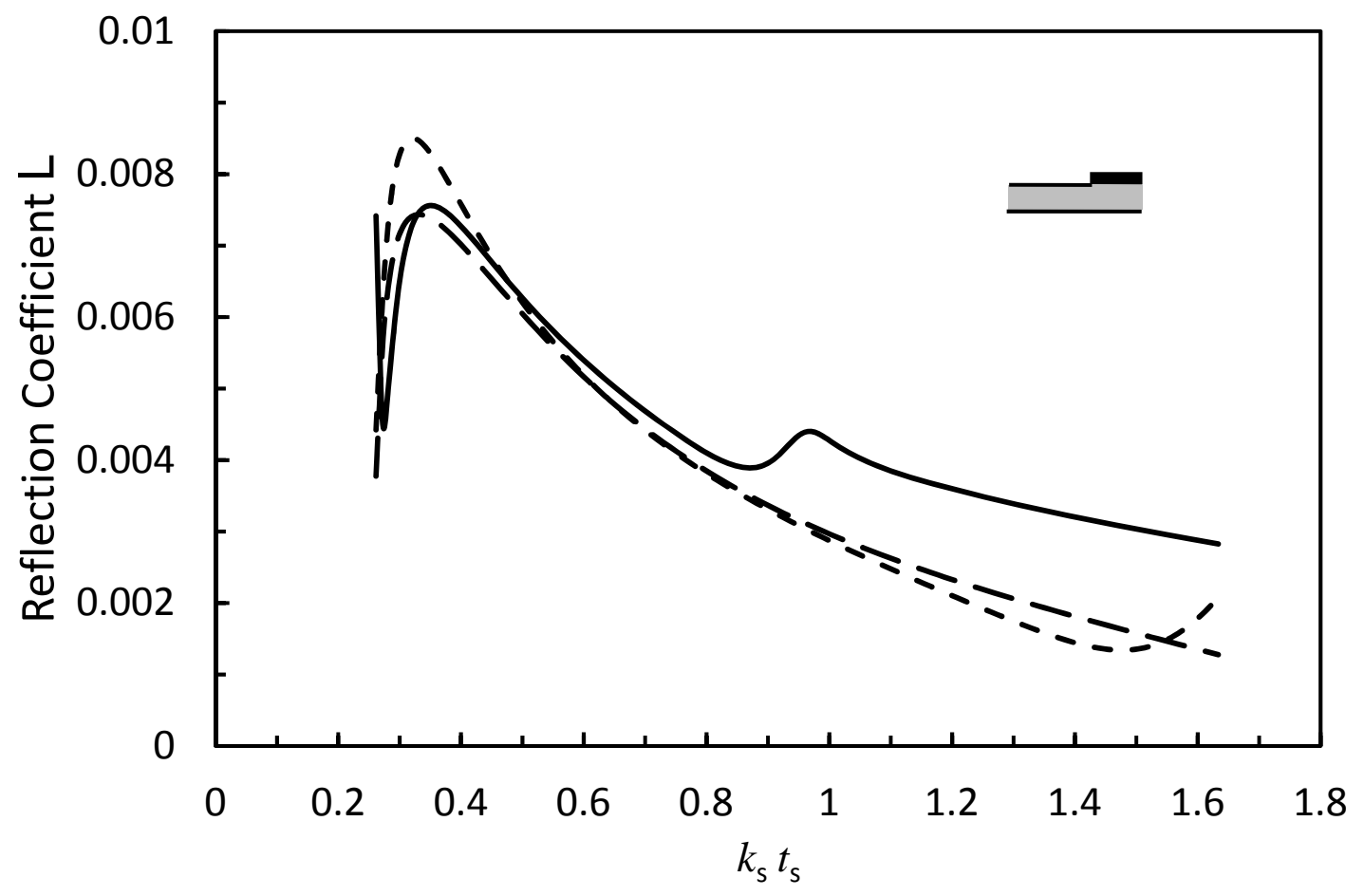

Figure 12. Mode matching predictions for the reflection coefficient of $L(0,2)$ for the transition between an uncoated and coated section of pipe:,$- t_{\mathrm{c}} / t_{\mathrm{p}}=1 ;----$,

$$
t_{\mathrm{c}} / t_{\mathrm{p}}=0.5 ; \quad---t_{\mathrm{c}} / t_{\mathrm{p}}=0.25
$$



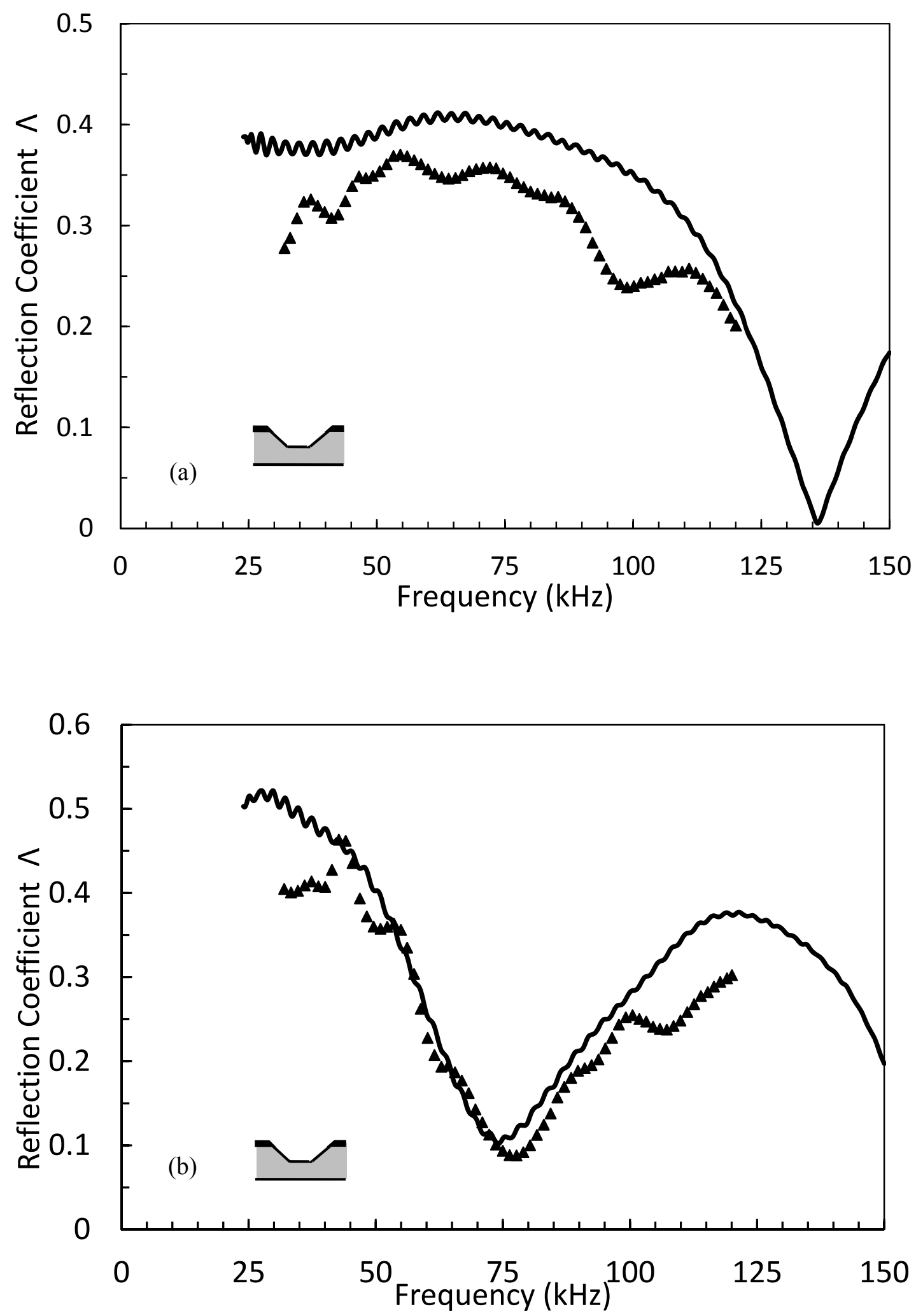

Figure 13. Hybrid method predictions for $\mathrm{L}(0,2)$ for a tapered defect in a coated pipe with $\gamma=$ $30^{\circ}$, (a) $L_{N}=15 \mathrm{~mm}$, (b) $L_{N}=30 \mathrm{~mm}$. — , prediction; $\boldsymbol{\Lambda}$, experiment. 